\title{
A fluid mechanical view on abdominal aortic aneurysms
}

\author{
VIRGINIE DUCLAUX ${ }^{1}$, FRANÇOIS GALLAIRE ${ }^{2}$, \\ AND CHRISTOPHE CLANET ${ }^{3} \dagger$ \\ ${ }^{1}$ IRPHE, UMR 6594, 49 rue F. Joliot-Curie, 13384 Marseille, France \\ ${ }^{2}$ LFMI, Ecole Polytechnique Fédérale de Lausanne, 1015 Lausanne, Switzerland \\ ${ }^{3}$ LadHyX, UMR 7646, Ecole Polytechnique, 91178 Palaiseau, France
}

(Received 22 September 2008; revised 7 July 2010; accepted 12 July 2010)

\begin{abstract}
Abdominal aortic aneurysms are a dilatation of the aorta, localized preferentially above the bifurcation of the iliac arteries, which increases in time. Understanding their localization and growth rate remain two open questions that can have either a biological or a physical origin. In order to identify the respective role of biological and physical processes, we address in this article these questions of the localization and growth using a simplified physical experiment in which water (blood) is pumped periodically (amplitude $a$, pulsation $\omega$ ) in an elastic membrane (aorta) (length $L$, crosssection $A_{0}$ and elastic wave speed $c_{0}$ ) and study the deformation of this membrane while decharging in a rigid tube (iliac artery; hydraulic loss $K$ ). We first show that this pulsed flow either leads to a homogenous deformation or inhomogenous deformation depending on the value of the non-dimensional parameter $c_{0}^{2} /\left(a L \omega^{2} K\right)$. These different regimes can be related to the aneurysm locations. In the second part, we study the growth of aneurysms and show that they only develop above a critical flow rate which scales as $A_{0} c_{0} / \sqrt{K}$.
\end{abstract}

Key words: biomedical flows, flow-vessel interactions

\section{Introduction}

\subsection{Definitions and history}

The use of the word aneurysm (dilate in Greek) in medicine seems to go back to Guy de Chauliac (1373), medical doctor of four popes and considered as the 'father' of mediaeval surgery. He used this word in La grande Chirurgie to designate the local dilatation of an artery. The study of aneurysms progressed with the development of medical techniques, starting with the stethoscope of Laennec (1819), who writes:

We use aneurysm to designate either the dilation of an artery or the presence of a sack connected to the artery. The first case is called true aneurysm by surgeons while the second is referred to as false aneurysm.

We learn from these definitions that aneurysms develop on arteries and can be classified in two different types which are now called fusiform ('true'), illustrated in figure 1(b) with an abdominal aortic aneurysm (AAA), and saccular ('false') illustrated

$\dagger$ Email address for correspondence: clanet@ladhyx.polytechnique.fr 


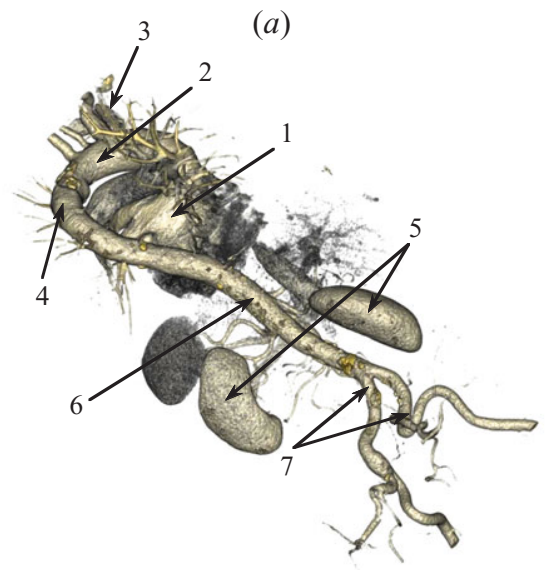

(b)

(c)
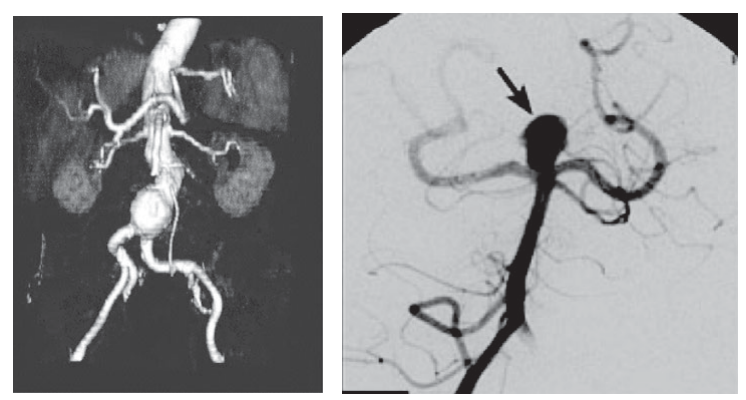

FIGURE 1. (Colour online) (a) Three-dimensional angiography of an aorta without aneurysm: (1) heart, (2) aortic arch, (3) coronary arteries, (4) thoracic aorta, (5) kidneys, (6) abdominal aorta and (7) iliac arteries. (b) Angiography of an aorta with an abdominal aortic aneurysm. (c) Angiography of a cerebral aneurysm.

in figure 1(c) with a cerebral aneurysm. We focus on fusiform aneurysms and use as a paradigm the AAA (Roberts 1959; Glagov 1961).

\subsection{Physiological characteristics}

The main geometrical features of the aorta are presented in figure 1(a): at the exit of the left ventricle, the aortic arch conducts the aorta from the heart to the spinal column. The thoracic and abdominal aortas designate the 'straight' portions of the aorta located above and below the diaphragm, respectively. At the end of the spinal column, the aorta splits into two iliac arteries, which irrigate each of the legs. For humans, the aorta is the longest artery $(L \approx 40 \mathrm{~cm})$ and we observe from this angiography that its diameter $(D \approx 2 \mathrm{~cm})$ is much larger than the secondary connecting vessels. To a first approximation, the aorta will thus be considered as a plain tube without branching until the iliac arteries.

We observe in figure $1(b)$ that the AAA develops above the bifurcation of the iliac arteries. According to recent studies (Lasheras 2007), AAA rarely appears in individuals under 50 years of age, but their incidence increases drastically at the age of 55 and peaks in the early 80s. A large screening study conducted in Norway in 1994-1995 showed that AAA are present in $8.9 \%$ of men and $2.2 \%$ of women over 60 years of age.

The exact reasons for the development of AAA are still unknown (Alexander 2004, McAuley et al. 2002). However, atherosclerosis and hypertension have been recognized as important risk factors. Atherosclerosis can be responsible for the alteration of the wall properties while hypertension can be responsible for the overpressure leading to a local deformation of the aorta.

With regard to the growth rate of the aneurysms and the risk of rupture, they both increase with the size of the aneurysm: the study by Guirguis \& Barber (1991), dedicated to the growth after 12 months of four classes of aneurysms (diameters less than $4 \mathrm{~cm}$, from 4 to $5 \mathrm{~cm}$, from 5 to $6 \mathrm{~cm}$ and more than $6 \mathrm{~cm}$ ), shows that they have grown of $0.2,0.3,0.4$ and $0.8 \mathrm{~cm}$, respectively. With regard to the risk of rupture, 
the same study reveals that the risk factor for aneurysms of diameters less than $4 \mathrm{~cm}$, from 4 to $5 \mathrm{~cm}$ and larger than $5 \mathrm{~cm}$ is 1,2 and $20 \%$, respectively.

\subsection{Physical properties and orders of magnitude}

The blood is composed of different cells, the larger ones being the leucocytes $\left(d_{\text {max }} \approx 22 \mu \mathrm{m}\right.$ in diameter) and the more numerous being the red blood cells (mean diameter $7 \mu \mathrm{m}$, Humphrey \& Delange 2004). Depending on the size $R_{0}$ of the vessel in which the blood is flowing, it can behave either as a Newtonian $\left(R_{0} / d_{\max } \gg 1\right)$ or as a non-Newtonian $\left(R_{0} / d_{\max }<1\right)$ liquid (Reinke 1986). In the limit of large vessels such as the aorta $\left(R_{0} / d_{\max } \approx 450\right)$, the blood behaves as a Newtonian liquid of kinematic viscosity $v \approx 4.10^{-6} \mathrm{~m}^{2} \mathrm{~s}^{-1}$ (Pedley 1980; Chandran \& Yearwood 1981; Ku 1997).

The non-intrusive velocity measurements in the human aorta obtained by Cheng et al. (2003) using the magnetic resonance imaging (MRI) reveal that the mean velocity is always smaller than the metre per second $\left(U \approx 0.8 \mathrm{~m} \mathrm{~s}^{-1}\right)$ and that the velocity profile is close to a top hat with thin boundary layers $O(1) \mathrm{mm}$.

The wall of arteries is composed of three layers, the intima, the media and the adventia. The media is composed of elastin, which enables the dilatation of the artery when exposed to a transmural pressure increase. For mammals, the order of magnitude of the pressure variation over a cardiac cycle is of the order of $\delta p \approx \rho g H$, where $H$ is the size of the mammal (McDonald 1960, 1968), which means that $\delta p \approx 10^{4} \mathrm{~Pa}$ for a human. The corresponding relative variations of the cross-section of the arteries are of the order of $10 \%: \delta A / A \approx 0.1$. To describe the elastic behaviour of the arteries, the physiologists use the distensibility $\mathscr{D} \equiv A^{-1} \delta A / \delta p$, which can be evaluated using the previous estimations to $10^{-5} \mathrm{~m}^{2} \mathrm{~N}^{-1}$ for a human (McDonald 1968; Fung 1990). Lighthill (1975) showed that this deformation can be localized and can propagate without dispersion along the aorta with the wave speed $c_{0}=1 / \sqrt{\rho \mathscr{D}} \approx 10 \mathrm{~m} \mathrm{~s}^{-1}$, much larger than the flow velocity.

To model the aortic flow in a laboratory experiment, one needs to respect the non-dimensional numbers which characterize the actual flow. For a Newtonian liquid flowing in a tube of radius $R_{0}$ with the mean velocity $U$, the flow is first characterized by the Reynolds number $R e \equiv U R_{0} / \nu \approx 10^{3}$, which compares inertial effects $\left(\rho R_{0}^{2} U^{2}\right)$ to viscous forces $\rho \nu U R_{0}$. In this limit of high Reynolds numbers, inertia dominates and viscous effects are concentrated in thin boundary layers close to the walls.

In the case of a pulsating flow (pulsation $\omega$ ), Stokes' second problem gives a scaling of the boundary layer size $\delta \approx \sqrt{v / \omega}$. The ratio $R_{0} / \delta$ gives the relative size of the boundary layer compared with the size of the tube. In physiological applications, this ratio is known as the Womersley number $W_{o} \equiv R_{0} \sqrt{\omega / v}$ (Womersley 1957). For the aorta, we evaluate $W o \approx 20$. The boundary layer is thus small compared with the size of the artery, and we recover the observation done considering the plug-like velocity profiles measured by Cheng et al. (2003).

Finally, since the artery is elastic, one needs to respect the ratio between the elastic wave propagation $c_{0}$ and the velocity of the flow $U$. This ratio $S h \equiv U / c_{0}$ is classically called the Shapiro number (Païdoussis 2006). For the aorta $S h \approx 0.1$.

In conclusion, in order to reproduce the physiological flow, the experiment must be conducted in the limit

$$
R e \gg 1, \quad W_{o} \gg 1, \quad S h \ll 1 \text {. }
$$

\subsection{Objectives}

Abdominal aortic aneurysms have been found to be systematically localized before the iliac bifurcation and are characterized by a growth rate which increases with their 


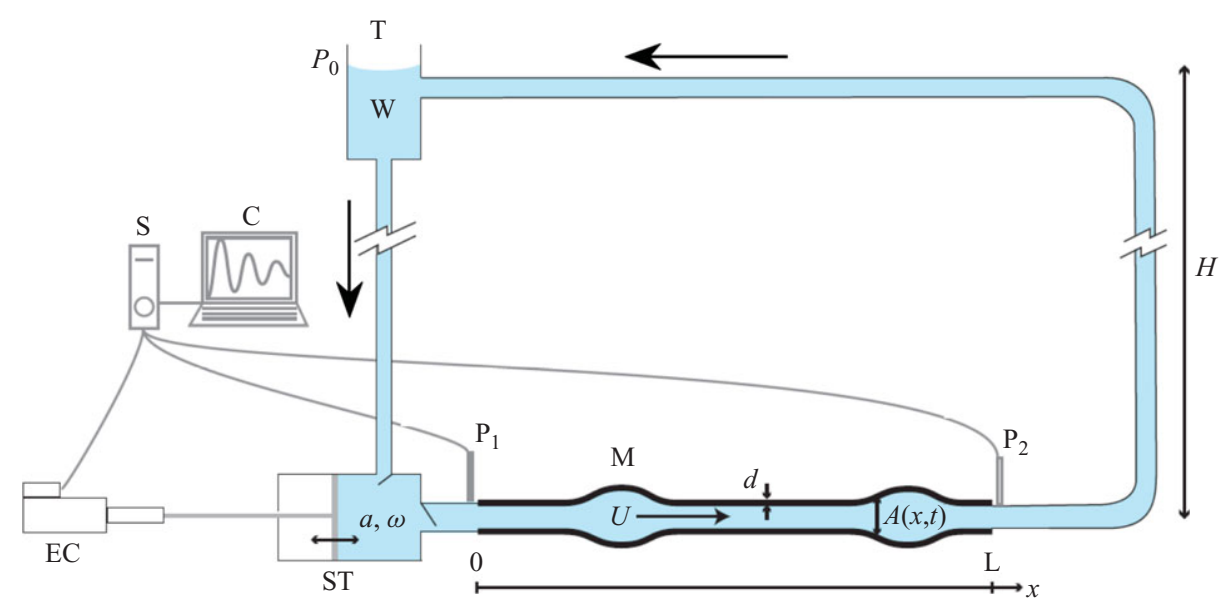

FIGURE 2. (Colour online) Sketch of the experiment: $\mathrm{T}=$ tank, $\mathrm{W}=$ water, $\mathrm{ST}=$ stroke (oscillatory motion of the amplitude $a$ and the angular frequency $\omega$ ), $\mathrm{C}=$ computer, $\mathrm{S}=$ servo, $\mathrm{EC}=$ electrical cylinder, $\mathrm{P}_{1}$ and $\mathrm{P}_{2}=$ pressure sensors and $\mathrm{M}=$ elastic membrane. We also use $d$ for the local membrane thickness, $U$ for the local flow velocity and $A$ for the local cross-section. Finally, $P_{0}$ stands for the atmospheric pressure and $H$ is the vertical distance between the membrane and the tank.

size and a characteristic period of development of the order of a year. To determine the respective role of biology and mechanics in AAA, we reproduce, in a scaled model, the pulsating flow of a Newtonian liquid in an elastic membrane and identify the mechanical conditions under which aneurysms can form.

The experimental set-up is presented in $\S 2$. The dynamics of the aorta on the time scale of the heart beat is presented in $\S 3$, where the question of the localization is addressed. The development and growth rate of the aneurysms is addressed in $\S 4$, prior to the conclusion in $\S 5$.

\section{Experimental set-up and protocol}

In this section, we first give an overview of the experiment and then detail each of the main parts before presenting the diagnostic tools used to quantify the dynamics of the deformation of the membrane.

\subsection{Overview}

The experimental set-up is sketched in figure 2: the water (blood) (W) is contained in a tank $(\mathrm{T})$ with a free surface maintained at the constant atmospheric pressure $P_{0}$. Using a computer (C) we move, via the servo (S), an electric cylinder (EC) which pumps the water in the stroke (heart) (ST) with the amplitude $a$ and the frequency $\omega$. We define the amplitude with respect to the liquid motion at the exit of the stroke, which means that the velocity of the liquid as it enters the membrane (aorta) (M) is of the order of $a \omega$. Two valves in the stroke impose the motion of the liquid in the anticlockwise direction as indicated by the arrows. Two pressure sensors (P1) and (P2) enable the measure of the pressure variation across the membrane. At the exit of the elastic membrane, the liquid returns to the tank via a rigid tube (iliac artery). The tank is located above the membrane, at a height $H \approx 1 \mathrm{~m}$ which imposes a mean pressure jump across the membrane of the order of $\rho g H$. This positive pressure jump ensures that as long as the velocity in the membrane remains smaller than $\sqrt{2 g H}$, 


\begin{tabular}{|c|c|c|c|c|c|}
\hline & $\begin{array}{c}L \\
(\mathrm{~cm})\end{array}$ & $\begin{array}{l}R_{0} \equiv \sqrt{A_{0} / \pi} \\
\quad(\mathrm{mm})\end{array}$ & $\begin{array}{c}d_{0} \\
(\mathrm{~mm})\end{array}$ & $\begin{array}{c}c_{0} \\
\left(\mathrm{~m} \mathrm{~s}^{-1}\right)\end{array}$ & $\begin{array}{c}E \\
(\mathrm{MPa})\end{array}$ \\
\hline $\begin{array}{l}\text { Human aorta } \\
\text { (Fung 1997; } \\
\text { Groenink et al. 1999) }\end{array}$ & 40 & $7.4-11.3$ & $\approx 1$ & $5.15 ; 6$ & $\begin{array}{l}\text { Elastin: } 2.4 \\
\text { Collagen: } 114 \\
\text { Global: } 5.3\end{array}$ \\
\hline $\begin{array}{l}\text { Latex membrane } \\
\text { Michelin }^{\circledR}\end{array}$ & $0-200$ & 9.5 & 0.7 & 6 & 1.07 \\
\hline $\begin{array}{l}\text { Human iliac artery } \\
\text { (Gray 1918; Li et al. 1981; } \\
\text { Medynsky et al. } 1998 \text { ) }\end{array}$ & 5 & $\approx 5.5$ & $\approx 0.7$ & $9 ; 11.5$ & Global: 10 \\
\hline
\end{tabular}

the membrane is always under tension. Since arteries work in this inflated regime, the whole study is conducted in this limit.

\subsection{An elastic membrane for the aorta}

As an elastic membrane, we have used latex tyre tubes from Michelin, the properties of which are presented in table 1 and compared with the properties of the human aorta and iliac arteries. The elastic membrane is characterized by its length $L$, initial unstretched cross-section $A_{0}$, initial thickness $d_{0}$ and Young modulus $E$. We also report in table 1 the value of the elastic wave speed $c_{0} \equiv \sqrt{d_{0} E / 2 \rho R_{0}}$ (Lighthill 1975), defined with $\rho$ as the density of the contained liquid and $R_{0} \equiv \sqrt{A_{0} / \pi}$ as the initial radius of the membrane.

Note in table 1 that the geometrical properties $\left(L, R_{0}, d_{0}\right)$ as well as the dynamical characteristic $\left(c_{0}\right)$ of the latex membrane are compatible with the characteristics of the human aorta reported in the literature. We also underline the higher rigidity of the iliac artery which is modelled in our experiment using the limit of a rigid tube (see §2.2.4).

\subsubsection{Experimental characterization of the elasticity of the membrane}

To characterize the elastic properties of the latex membrane, we have used an hydraulic traction machine, presented in figure $3(a)$ : a rectangular piece of material (length $L_{0}$, width $b_{0}$ and thickness $h_{0}$ ) is initially clamped between cylindrical jaws. A controlled traction force $F$ is then exerted via the jaws on the membrane and the corresponding equilibrium length $L$ is measured. The relation between the stress $\sigma \equiv F /(h b)$ and the relative extension $\epsilon \equiv\left(L-L_{0}\right) / L_{0}$ is presented in figure $3(b)$. In this graph, the force $F$ and the length $L$ are measured while the cross-section $h b$ is deduced from the initial cross-section $h_{0} b_{0}$ via the incompressibility relation $h b=h_{0} b_{0} L_{0} / L$, which assumes a Poisson coefficient $v_{p}=1 / 2$.

In the limit of small relative deformation $\epsilon<2$, the stress is proportional to the deformation $\sigma \approx 10^{6} \epsilon$. This is the Hookean regime from which one deduces the value of the Young modulus $E \approx 1 \mathrm{MPa}$ presented in table 1 . For a higher relative deformation, the stress is a stronger function of the deformation as plastic effects appear (Carpenter \& Pedley 2003).

\subsubsection{A pressure law for the cylindrical membrane}

Let us now consider a cylindrical membrane such as the one presented schematically in figure $4(a)$. When there is no pressure jump across the membrane $\left(P=P_{0}\right)$ the 

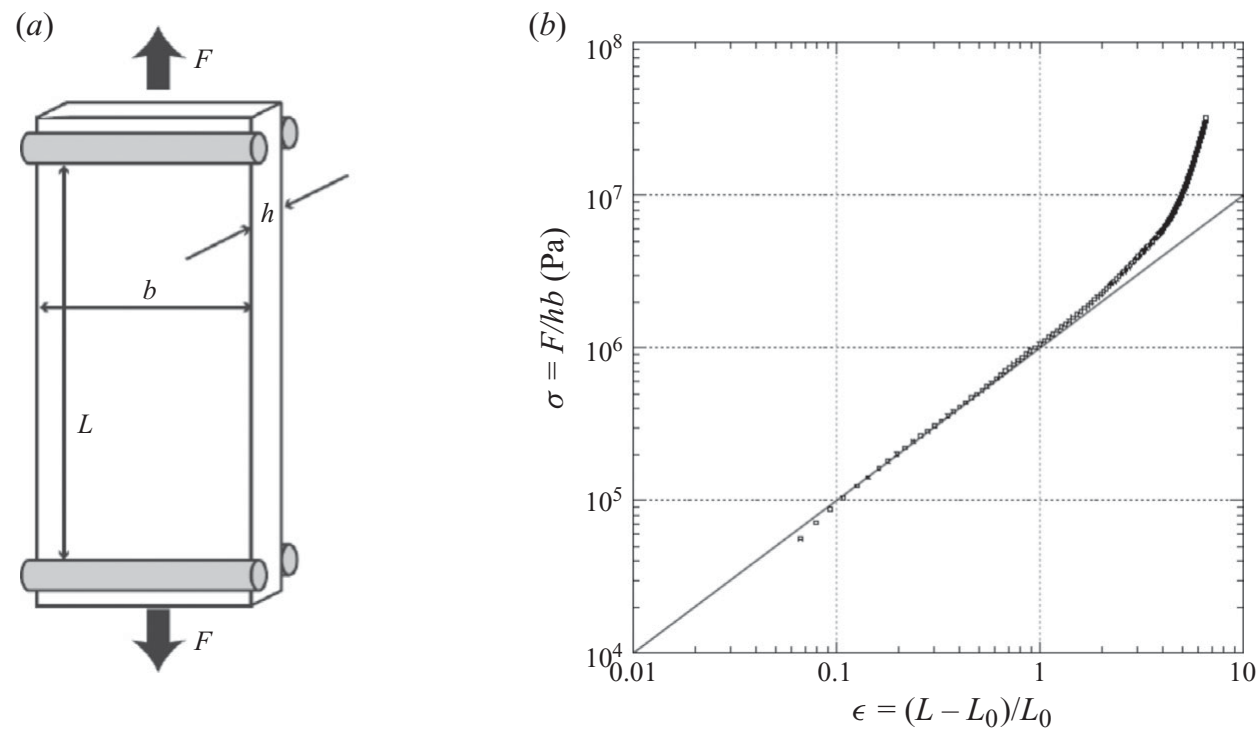

FIGURE 3. Characterization of the elastic properties of the membrane: $(a)$ scheme of the traction experiment, $(b)$ results obtained for the traction stress $\sigma \equiv F /(h b)$ as a function of the relative elongation $\epsilon \equiv\left(L-L_{0}\right) / L_{0}$.

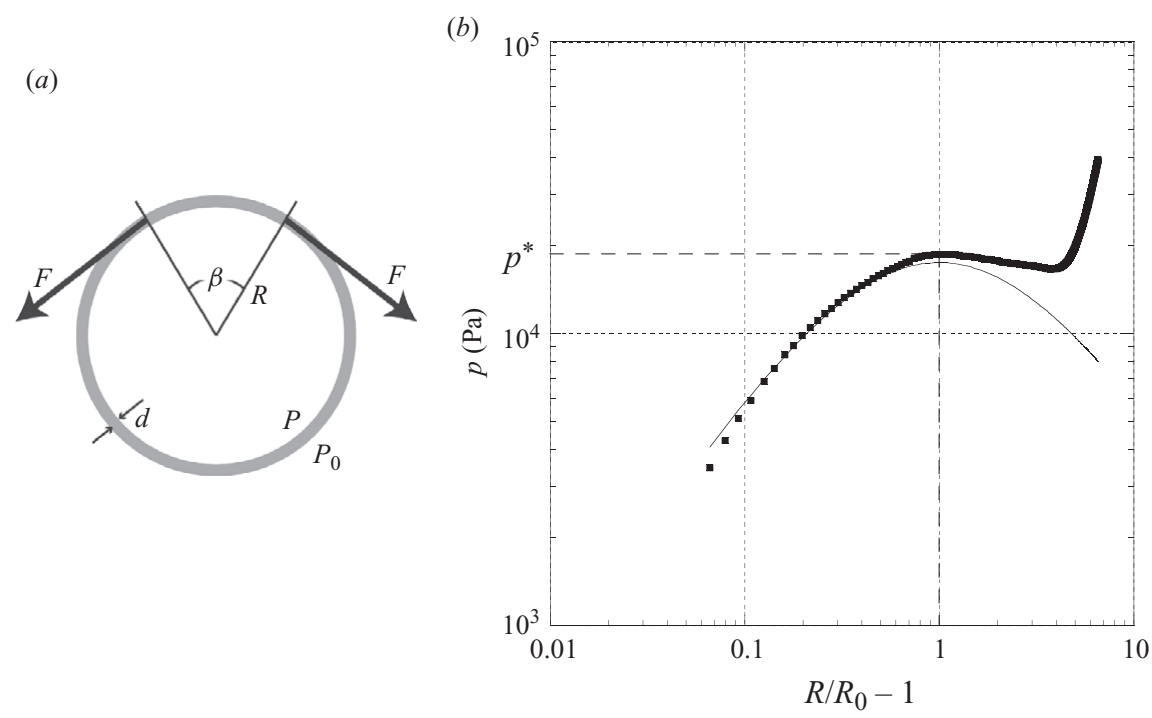

FIGURE 4. A pressure law for the cylindrical membrane: $(a)$ scheme for the equilibrium, $(b)$ experimental evolution of the transmural pressure $p$ with the relative deformation $R / R_{0}-1$ (घ). The solid line corresponds to the theoretical curve (2.2).

radius of the membrane is $R_{0}$. When the pressure across the membrane increases $p \equiv P-P_{0}>0$, the new equilibrium radius $R$ results from the balance between the pressure and elastic stresses. For a small element of the angular extension $\beta$, this equilibrium reads

$$
p=\frac{d_{0}}{R_{0}} \frac{\sigma(\epsilon)}{(1+\epsilon)^{2}},
$$




\begin{tabular}{lccccc}
\hline & $f$ & $\begin{array}{c}\text { Ejected volume } \Omega \\
\left(\mathrm{cm}^{3} \mathrm{cycle}^{-1}\right)\end{array}$ & $\begin{array}{c}\text { Reynolds no. } \\
R e=U R_{0} / v\end{array}$ & $\begin{array}{c}\text { Womersley no. } \\
W o=R \sqrt{\omega / v}\end{array}$ & $\begin{array}{c}\text { Shapiro no. } \\
S h=U / c_{0}\end{array}$ \\
Human heart & $1.25-3$ & $\sim 80$ & $700-2500$ & $\sim 20$ & $0.1-0.25$ \\
Electric cylinder & $0.1-10$ & $5.6-168$ & $900-10^{4}$ & $7-40$ & $0.1-0.16$
\end{tabular}

TABLE 2. Properties of the human heart (Shapiro 1977; Chandran \& Yearwood 1981; Fung 1990; Ku 1997; Groenink et al. 1999; Humphrey \& Delange 2004) and the present experiment.

where $\epsilon \equiv R / R_{0}-1$. The experimental evolution $p\left(R / R_{0}\right)$ is shown in figure $4(b)$ by solid squares. At small relative extensions $(\epsilon<1)$, the radius of the membrane increases with the pressure. This evolution stops at $R=2 R_{0}$, where the pressure reaches a maximum $p^{\star} \approx 18500 \mathrm{~Pa}$. If the pressure is increased above this critical pressure, one observes a jump on the radius from $R=2 R_{0}$ to $R \approx 6 R_{0}$. For an even higher pressure, since the membrane becomes stiffer, the radius increases with a lower sensitivity to the pressure.

Using the Hookean expression of the stress $\sigma=E \epsilon$, the pressure-radius equation (2.1) reduces to

$$
p=E \frac{d_{0}}{R_{0}} \frac{\epsilon}{(1+\epsilon)^{2}} .
$$

This relation is shown by a thin solid line in figure $4(b)$. The comparison with the experimental data shows that the linear Hookean approximation does capture the evolution of the pressure up to the pressure maximum $p^{\star}$. It predicts correctly the extension at which the maximum occurs $\left(\mathrm{d} p / \mathrm{d} R=0\right.$ when $\left.R / R_{0}=2\right)$ as well as the absolute value of the critical pressure. Since the Hookean model does not account for the stiffening of the membrane at large relative deformations (observed in figure $3 b$ ), it fails to describe the increase of the pressure observed in the range $\epsilon>5$. However, since the whole study is conducted in the range of deformations $R / R_{0} \leqslant 2$, we will use the Hookean approximation (2.2) to describe the pressure-radius relation. In terms of the cross-section $A$, this relation can be rewritten

$$
p(A)=2 \rho c_{0}^{2}\left(\sqrt{\frac{A_{0}}{A}}-\frac{A_{0}}{A}\right) .
$$

Under this form, the relation has already been used (Paquerot \& Lambrakos 1994) and discussed in Olsen \& Shapiro (1967). In Olsen \& Shapiro (1967), the authors prefer a pressure law derived from statistical physics given by $p=1 / 2 \rho c_{0}^{2}\left[1-\left(A_{0} / A\right)^{2}\right]$. Both expressions are equal in the limit $A / A_{0}=1+\epsilon_{A}$, where $p=\rho c_{0}^{2} \epsilon_{A}\left(\epsilon_{A} \ll 1\right)$. However, the essential difference between the two expressions is that one predicts a maximum (see (2.3)) while the other predicts a saturation. Since the maximum and the corresponding instability is observed experimentally as shown in figure $4(b)$ we have decided to work with the Hookean model (2.3).

\subsection{An electrical cylinder for the heart}

Concerning the heart, we report in table 2 its main mechanical characteristics: beat frequency $f \equiv \omega / 2 \pi$ and ejected volume per beat $\Omega$. These values are compared with those obtained artificially from the electric cylinder (IDC-Motion EC3-B23) controlled with a computer. Both the frequency and the ejected volume range of the physiological system are covered by the experimental counterpart. These parameter 
(a)

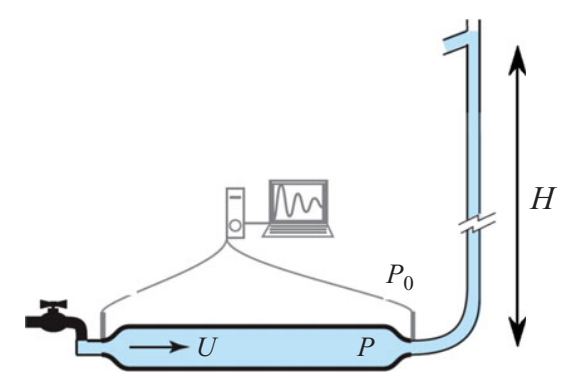

(b)

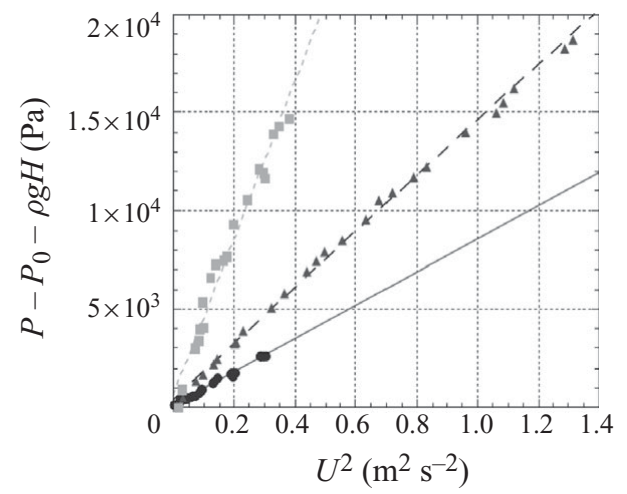

FIGURE 5. (Colour online) Resistance to the flow: $(a)$ scheme of the experimental set-up dedicated to the description of the resistance, $(b)$ measurement of the pressure in the membrane $\Delta p \equiv P-P_{0}-\rho g H$ as a function of the square of the mean velocity $U^{2}$. The proportionality $\Delta p=K \rho U^{2}$ is illustrated for three different rigid tubes which lead to $K=8(\bullet), K=14(\triangle)$ and $K=40(\square)$.

ranges have been chosen to generate a flow in the elastic membrane in close similarity with that observed in the aorta.

The similarity between both flows is achieved by using similar non-dimensional numbers: Reynolds, Womersley and Shapiro numbers. Their typical range of variations is indicated in table 2 . The Reynolds number $R e \equiv U R_{0} / \nu=\Omega f / \pi R_{0} \nu$ is typically $O\left(10^{3}\right)$ in the human aorta (Chandran \& Yearwood 1981; Fung 1990; Groenink et al. 1999) whereas the range of the experiment is $900-10^{4}$. The Womersley number $W o \equiv R_{0} / \sqrt{v / \omega}$ is $O(20)$ in the aorta (Fung 1990; Ku 1997; Humphrey \& Delange 2004) and ranges between 7 and 40 in our experiment. Finally, the Shapiro number $S h \equiv U / c_{0}$ compares the velocity of the flow to the propagation velocity of the elastic waves on the aorta. The Shapiro number is small in the aorta (typically 0.1 , Pedley 1980) and the same range is achieved in our experiment.

\subsection{A rigid tube as iliac artery}

As seen in table 1 and mentioned in $\S 2.2$, the iliac arteries are stiffer than the aorta. Moreover, the network of subsequent arteries and vessel behaves as a 'resistance' for the flow in the aorta (McDonald 1960). To model these two effects, we connect the elastic membrane to a rigid tube the diameter (and length) of which is used to change the 'resistance' to the flow. Experimentally, this connection is achieved using a hose clamp.

In analogy with Ohm's law, the resistance is classically defined as the relation between the pressure and the velocity in the membrane.

To measure this resistance, we have conducted a specific experiment presented in figure 5(a): first, the elastic membrane is filled with water and set into tension by a static pressure $P_{s} \equiv P_{0}+\rho g H$ imposed by the rigid tube which connects the membrane to an overflow standing at a height $H$ above the elastic tube. A mean velocity $U$ is then imposed and we measure the corresponding pressure difference $\Delta p \equiv P-P_{s}$. The evolution $\Delta p(U)$ is presented in figure 5(b). We observed a linear relation between the pressure difference and the square of the mean velocity: $\Delta p=K \rho U^{2}$. The constant of proportionality $K$ changes from 8 to 14 and then 40 as we have varied the rigid tube length and diameter. Since the Reynolds number of the flow in the experiment 
(a)

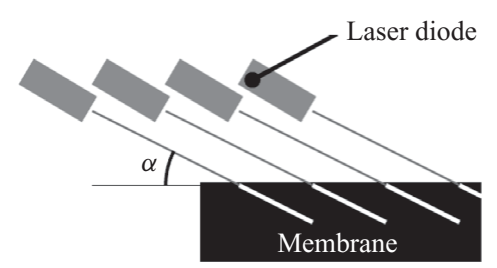

(b)

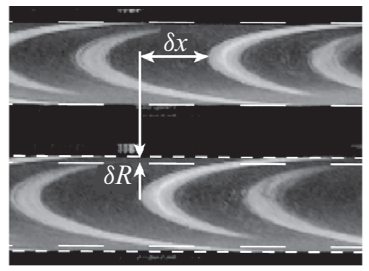

(c)

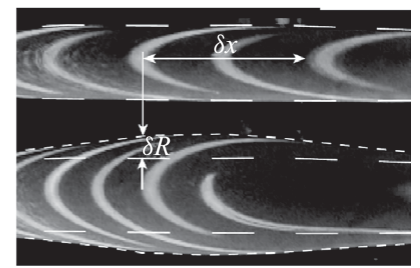

FIGURE 6. Experimental method used to measure the deformation of the membrane: $(a)$ scheme of the set-up, $(b)$ example of the observation in the case of a homogeneous deformation, (c) example of the observation in the case of an inhomogeneous deformation.

is large $\left(>10^{3}\right)$, one is not surprised to obtain such a relationship for the resistance (Prandtl \& Tietjens 1957).

Furthermore, with a mean velocity of the order of the metre per second, the corresponding pressure losses for the three different resistances are 8000, 14000 and $40000 \mathrm{~Pa}$. These values are comparable to the pressure loss measured in a human aorta, $\approx 10^{4} \mathrm{~Pa}$ (McDonald 1960).

\subsection{Experimental methods}

In the limit of small deformations, the law of the membrane (2.3) shows that the relative deformation $\delta R / R_{0}$ is related to the pressure fluctuations $\delta p$ via the relation $\delta R / R_{0} \sim \delta p / \rho c_{0}^{2}$. The evaluation $\delta p \sim \rho U^{2}$ leads to $\delta R / R_{0} \sim\left(U / c_{0}\right)^{2} \approx 1-10 \%$. For a centimetric elastic membrane $\left(c_{0} \approx 6 \mathrm{~m} \mathrm{~s}^{-1}\right)$ and a typical metre per second flow, we deduce that the radial deformations are millimetric. Since the length of the aorta is of the order of $1 \mathrm{~m}$, the ratio between the length scale of the domain and the length scale of the deformation is $10^{3}$. This is out of the scope of a classical video camera $\sim 10^{2}$. We thus use a geometrical magnification to reduce the scale ratio to $10^{2}$ in order to observe the dynamical deformations of the membrane with a video camera. This geometrical zoom is presented in figure $6(a)$ : it consists of a comb of laser sheets inclined of an angle $\alpha$ with respect to the membrane. As shown in figure $6(b, c)$, each laser sheet intersects the cylindrical membrane and produces a bright elliptical trace. In figure $6(b, c)$, the image at the top represents the membrane prior to the deformation (the edges of the membrane are marked by long dashed lines) while the image at the bottom presents the membrane after the deformation (the edges of the membrane after the deformation are underlined by short dashed lines). The radial deformation being millimetric $\delta R \approx 1 \mathrm{~mm}$, we observed that it induces a displacement of the laser trace $\delta x \approx 1 \mathrm{~cm}$. The geometrical relation between the two variations is $\delta x=2 \delta R / \tan \alpha$. The displacements are reduced to a translation in the case of a homogeneous deformation (figure $6 b$ ) or lead to a more complex figure in the case of an inhomogeneous deformation (figure $6 c$ ).

\section{Dynamics on the time scale of the heart beat}

In this section, we consider the dynamics of the deformation of the elastic membrane on the time scale of a few periods of forcing. We first present the experimental results and then the model and numerics are developed to account for the observations. 
(a)

(b)

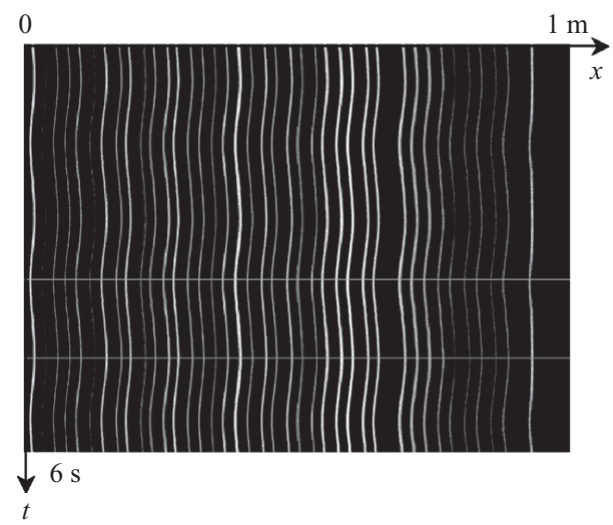

(c)

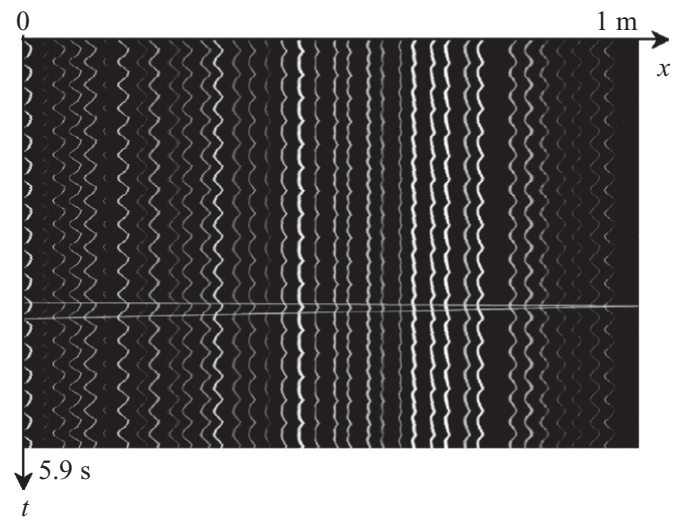

FIGURE 7. Spatio-temporal diagrams: $(a)$ view from above of the whole membrane $L=1 \mathrm{~m}$, $R_{0}=1 \mathrm{~cm} ;(b)$ spatio-temporal diagram obtained during $6 \mathrm{~s}$ at the frequency $f=0.5 \mathrm{~Hz}$ with the amplitude $a=270 \mathrm{~mm} ;(c)$ spatio-temporal diagram obtained during $5.9 \mathrm{~s}$ at the frequency $f=3 \mathrm{~Hz}$ with the amplitude $a=45 \mathrm{~mm}$.

\subsection{Experimental evidence of two distinct regimes}

The experimental results presented in this section have all been obtained with the resistance (of the iliac artery): $K=8$. In order to describe the time evolution of the deformation of the membrane, we use spatio-temporal diagrams: starting from a global view of the membrane such as the one presented in figure $7(a)$ we extract the centred horizontal line and construct a new image where its different lines correspond to the selected line at different times equally spaced. An example of such a spatio-temporal diagram is presented in figure $7(b)$. This diagram extends over $6 \mathrm{~s}$ and corresponds to the experimental conditions: $f=0.5 \mathrm{~Hz}$, amplitude $a=270 \mathrm{~mm}$ (velocity $a \omega=0.85 \mathrm{~m} \mathrm{~s}^{-1}$ ). At a given spatial location we observe that the deviation of the light oscillates in time with the period of $\approx 2 \mathrm{~s}$. We also measure an amplitude of the deviation of $\approx 1 \mathrm{~cm}$ which implies a radius variation $\delta R \approx 1 \mathrm{~mm}$.

Considering the spatial evolution of the deviations, figure $7(b)$ presents a synchronous translation of the different beams: the membrane thus undergoes an homogeneous deformation. Let us now consider the spatio-temporal diagram shown in figure $7(c)$ obtained at the same velocity $\left(0.85 \mathrm{~m} \mathrm{~s}^{-1}\right)$ but with different forcing conditions: $f=3 \mathrm{~Hz}$ and $a=45 \mathrm{~mm}$. The time evolution of the light deviation at a given location contains more harmonics than in figure $7(b)$. Furthermore, instead of a synchronous translation of the beams, we observe strong oscillations at the entrance and the exit of the membrane and almost no oscillations at an intermediate position $x \approx 0.6 \mathrm{~m}$. We also observe that the oscillations at the two ends oscillate out of phase.

We retain from the qualitative analysis of these spatio-temporal diagrams that depending on the value of the forcing parameters, the membrane can undergo either an homogeneous deformation or an inhomogeneous one.

The transition between the two regimes of deformation illustrated in figures $7(b)$ and $7(c)$ can be quantified. First, keeping the length of the membrane constant $(L=50 \mathrm{~cm})$, figure 8(a) shows the evolution of the critical amplitude of forcing $a^{\star}$ with the frequency $f^{\star}$. As the frequency increases from 0.65 to $1.5 \mathrm{~Hz}$, the critical 

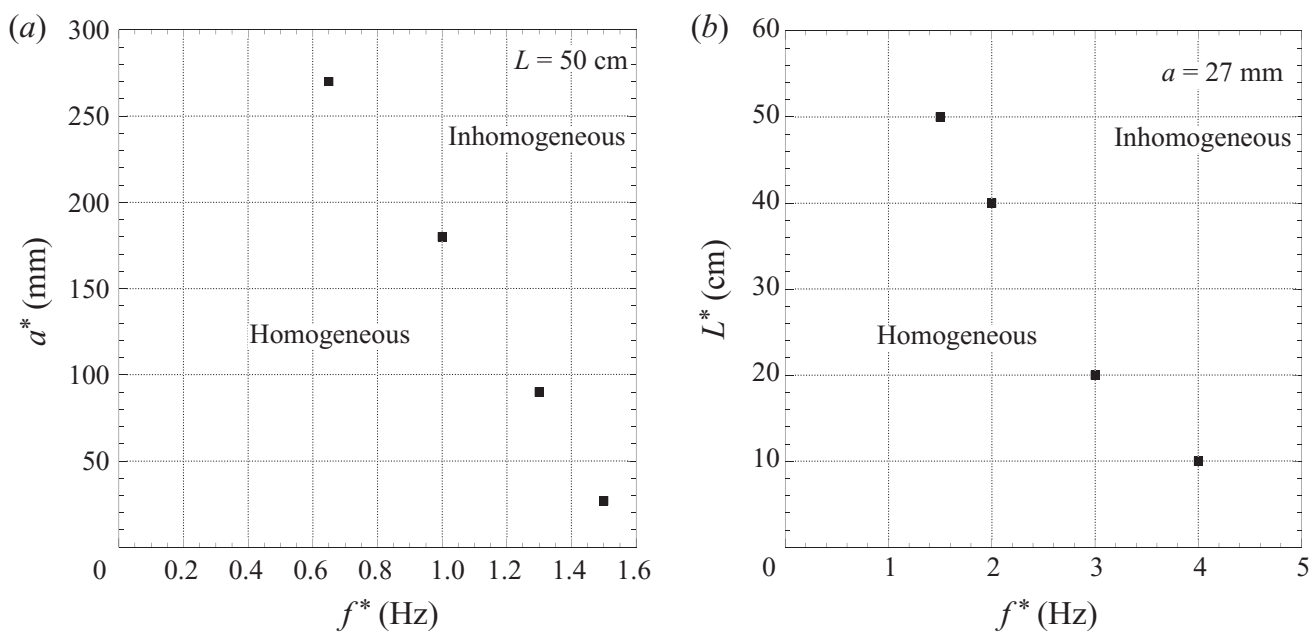

Figure 8. Quantitative analysis of the transition between the homogeneous and inhomogeneous deformation regimes: $(a)$ evolution of the critical amplitude $a^{\star}$ (denoted by solid squares) with the frequency $f^{\star}$ for a fixed length of the membrane $L=50 \mathrm{~cm} ;(b)$ evolution of the critical length $L^{\star}$ (denoted by solid squares) as a function of the frequency $f^{\star}$ for a fixed amplitude $a=27 \mathrm{~mm}$.

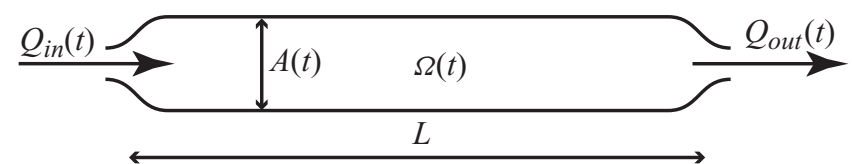

FIGURE 9. Windkessel model: $Q_{\text {in }}(t)$ is the inlet flow rate, $Q_{\text {out }}(t)$ is the outlet flow rate, $\Omega(t)$ is the volume of the fluid contained in the membrane and $A(t)$ is the mean cross-section.

amplitude above which an inhomogeneous membrane is observed decreases from 270 to $27 \mathrm{~mm}$.

Now keeping the amplitude of forcing constant $(a=27 \mathrm{~mm})$, figure $8(b)$ displays the evolution of the critical length $L^{\star}$ as a function of the forcing frequency $f^{\star}$. At a given frequency, we observe an homogeneous deformation on 'short' membranes and an inhomogeneous deformation above $L^{\star}$. As $f^{\star}$ increases from 1.5 to $4 \mathrm{~Hz}$, the critical length decreases from 50 to $10 \mathrm{~cm}$.

\subsection{Model}

To discuss the homogeneous character of the deformation, we first consider the Windkessel model (Frank 1905) which consists of a lumped (space averaged) model of the flow in the elastic membrane as shown in figure 9. In this limit, the cross-section of the membrane only depends on time through mass conservation which reads

$$
L \frac{\mathrm{d} A}{\mathrm{~d} t}=Q_{\text {in }}(t)-Q_{\text {out }}(t) .
$$

In this equation, $Q_{\text {in }}(t)$ is imposed by the 'heart' while $Q_{\text {out }}(t)$ is related to the pressure $p$ in the membrane via the relation

$$
Q_{\text {out }}(t)=A_{\text {out }} \sqrt{\frac{p}{K \rho}},
$$


where $A_{\text {out }}$ is the outlet cross-section of the elastic tube. The transmural pressure is given by the elastic membrane law (2.3). To estimate the characteristic time of deformation of the membrane, we consider a small deformation $A(t)=A_{0}[1+\epsilon(t)]$ and linearize (2.3), yielding

$$
\frac{\mathrm{d} \epsilon}{\mathrm{d} t}=\frac{Q_{i n}(t)}{L A_{0}}-\frac{c_{0}}{\sqrt{K} L} \sqrt{\epsilon} .
$$

When the inflow is steady $Q_{i n}(t)=Q_{i n}$, the system reaches the equilibrium $\epsilon_{e q}=\left(Q_{i n}^{2} K\right) /\left(A_{0}^{2} c_{0}^{2}\right)$ with the characteristic time $\tau_{e q}=L Q_{i n} K / A_{0} c_{0}^{2}$.

In the presence of a time-dependent periodic inflow rate $Q_{i n}(t)=A_{0} a \omega f(t)$ of the period $\tau_{f} \sim 1 / \omega$, the above analysis only holds if $\tau_{e q}<\tau_{f}$ (quasi-steady limit). Since $\tau_{e q}$ scales as $L$ there is always a portion of the size $L_{H}$ which satisfies this criterion:

$$
L_{H}=\frac{\tau_{f} c_{0}^{2}}{a \omega K},
$$

where $L_{H}$ is the length over which the membrane deforms homogeneously. To discuss the homogeneous/inhomogeneous transition observed on the whole membrane, $L_{H}$ must be compared with the total length $L$. Note that $L / L_{H}>1$ will lead to inhomogeneities while $L / L_{H}<1$ ensures a homogeneous deformation. The transition is thus described by the criterion

$$
\frac{a \omega^{2} K L}{c_{0}^{2}} \sim 1
$$

For a given membrane, this criterion predicts that the frequency $f^{\star}$ above which the membrane becomes inhomogeneous decreases as the amplitude of the forcing increases. For a fixed amplitude, it also implies that the critical length decreases with increasing forcing frequency. These two behaviours are qualitatively compatible with the experimental results presented in figure 8 .

More quantitatively, we present in figure 10 the evolution of the ratio $\left(\omega / c_{0}\right)^{2}$ as a function of $a L$ obtained for a constant resistance $K=8$. We observe in this figure that the data obtained from different tubes length, forcing amplitudes and frequencies collapse on a single curve and closely follow the criterion (3.5) shown by a solid line. Figure 10 enables us to establish the transition at $a \omega^{2} K L / c_{0}^{2} \approx 0.5$.

In the present model, since the Shapiro number is small $\left(a \omega / c_{0} \ll 1\right)$ we have neglected the role of waves, which could also be responsible for inhomogeneity. However, for linear elastic waves (Lighthill 1975) the wavelength $\lambda=2 \pi c_{0} / \omega$ is always larger than the membrane length. The numerical model developed in $\S 4.4$ accounts for the role of nonlinear waves (Pedley 1980).

\subsection{Physiological implications}

In this section, we propose some qualitative ideas on the location of aneurysms based on the two types of deformations observed from our elastic membrane. In the human, as in our experiments, the 'acoustic' wavelength $\lambda=2 \pi c_{0} / \omega\left(c_{0} \approx 5 \mathrm{~m} \mathrm{~s}^{-1}\right)$ is much larger than the length of the aorta $L=0.4 \mathrm{~m}$. The criterion (3.5) can be used to discriminate whether an artery submitted to the heart pulsating flow undergoes an homogeneous or inhomogeneous deformation. Using the values $K \approx 10$ (McDonald 1960 ) and $a \omega \approx 1 \mathrm{~m} \mathrm{~s}^{-1}$ (Cheng et al. 2003), inhomogeneous deformations are assumed to occur when $a L \omega^{2} K / c_{0}^{2}>0.5$. For the aorta, this ratio can be evaluated to 0.7. The aorta should thus undergo inhomogeneous deformations as shown in figure 11(a1). Assuming that inhomogeneous deformations induce, in time, inhomogeneous tissue 


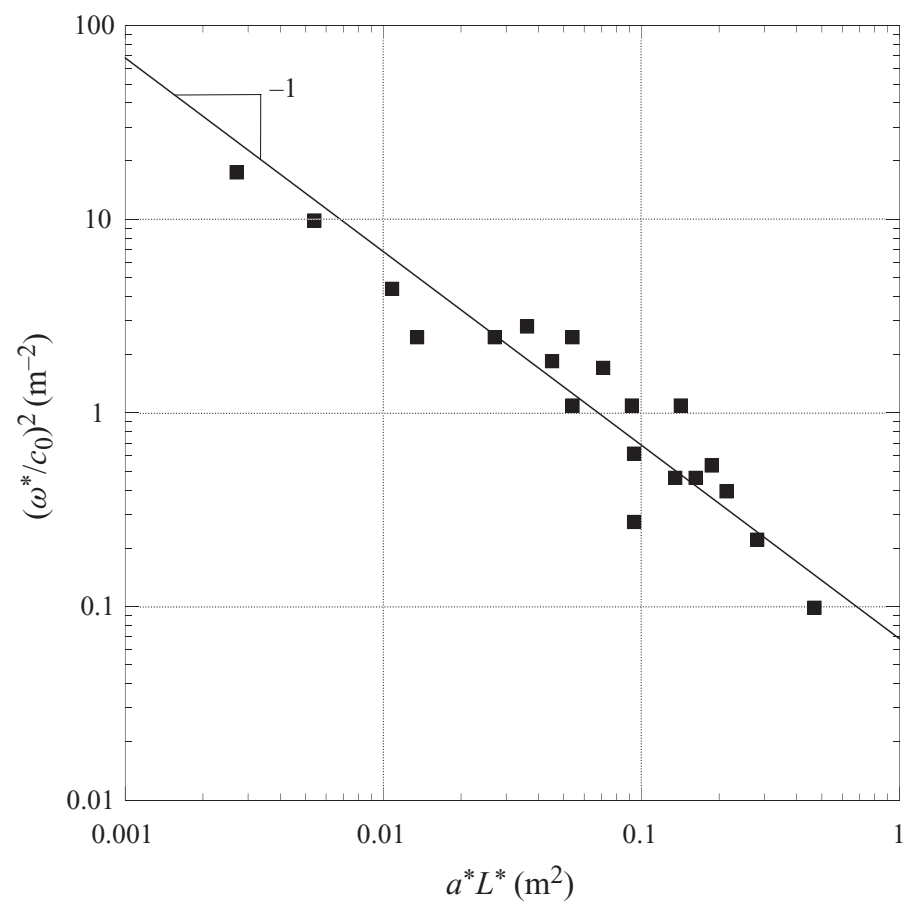

FIGURE 10. Transition observed experimentally between the synchronous and asynchronous regimes. The solid line stands for the behaviour expected from the scaling law (3.5).

(a)

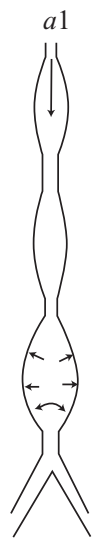

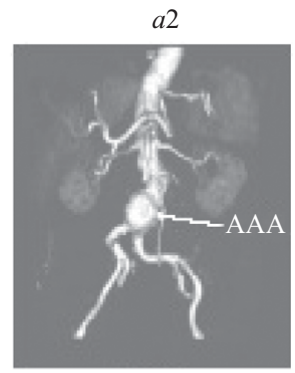

(b)

$b 1$

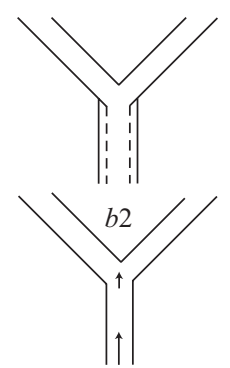

$b 3$

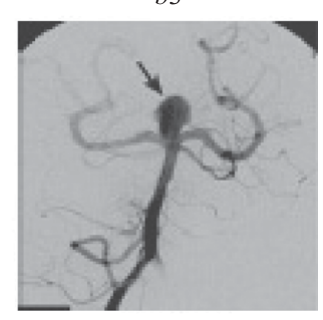

FIGURE 11. (a) Inhomogeneous deformation of a 'long artery', $(b)$ homogeneous deformation of a 'short artery'.

characteristics, one can understand at least qualitatively the formation of aneurysms at specific places along the vessel. The influence of the inhomogeneity of the deformation on the development of aneurysms is further studied with the numerics in $\S 4.4$.

For a shorter and stiffer artery such as the carotid artery $\left(L \approx 10 \mathrm{~cm}, c_{0} \approx 10 \mathrm{~m} \mathrm{~s}^{-1}\right)$, the situation is different: the parameter $a L \omega^{2} K / C_{0}^{2}$ is $O(0.01)$, which is smaller than the transition value 0.5 . The carotid artery should thus undergo homogeneous deformations as shown in figures $11(b 1)$ and $11(b 2)$. If the artery undergoes homogeneous deformation, the properties of the tissue should remain homogeneous 


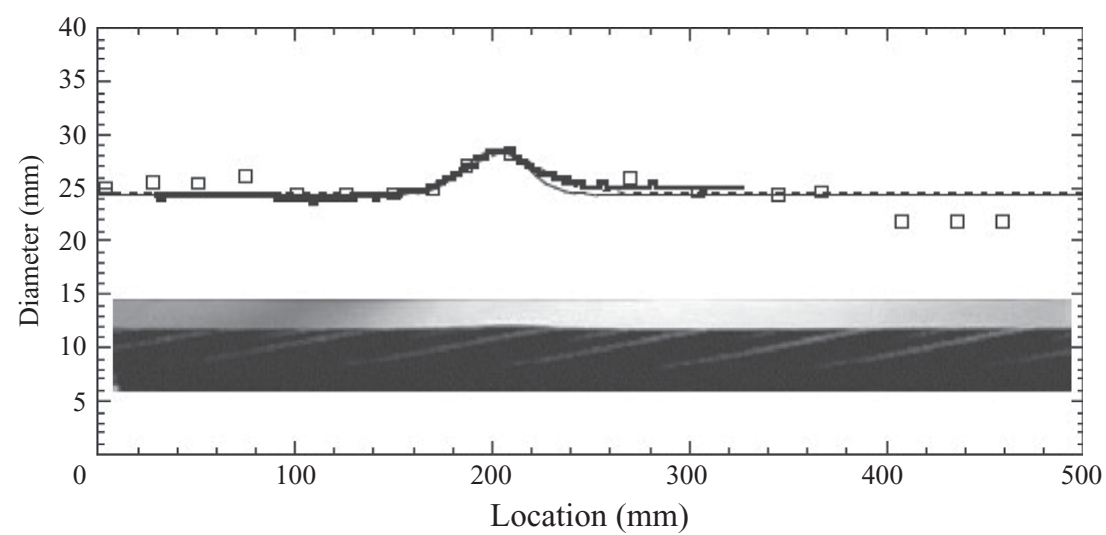

FIgURE 12. Diameter $(\mathrm{mm})$ of the membrane as a function of the position $x$ along the membrane, obtained by two simultaneous experimental diagnostics: a lateral close view delivered by a digital camera (reproduced in the figure inlet), and a set of discrete data points retrieved from the view from above of the displacement of the laser sheet device. Both shapes are well fitted by Gaussian functions.

and there is no reason for the appearance of an inhomogeneity along the artery. In this limit, if an inhomogeneity appears, it should be observed 'outside' the artery. The aneurysm presented in figure 11(b3) can be seen as an aneurysm developing outside an artery.

\section{Dynamics on the time scale of life}

\subsection{Experimental observations}

The time required for the development of an AAA is of the order of years and, therefore, of the order of millions of cardiac cycles. The aim of this section is to analyse if similar growing processes can be identified in our experiment and under which circumstances saturation is encountered or catastrophic growth (aneurysm) triggered.

In order to detect and characterize growing bulges on the membrane and to measure the precise membrane shape as a function of time and space, the laser sheet device described in $\S 2$ is used.

When a small deformation occurs as in the case shown in figure 12, the discrete set of data points is fitted by a Gaussian function of the form $D(x, t)=D_{b}(t)+$ $\Delta_{D}(t) \exp \left[-\left(x-x_{c}(t)\right)^{2} / \delta(t)^{2}\right]$, where $D_{b}(t)$ is the base diameter away from the bulge, $\Delta_{D}(t)$ is the bulge amplitude, $x_{c}(t)$ is the bulge centre position, and $\delta(t)$ is the bulge width. An alternative determination is obtained through a fit of a lateral close view delivered by a digital camera, as seen in the inset of figure 12, where the two fits are shown in the same graph at a given time. Both fitting functions agree to a certain extent, $24.5+3.64 \exp \left[-(x-205.45)^{2} / 820\right]$ for the imaging technique and $24.32+4.29 \exp \left[-(x-201.79)^{2} / 490\right]$ for the best fit over the laser deviations. These discrepancies are related not only to the experimental techniques but also to the presence of the bottom wall which breaks the axisymmetry. In this example, the relative discrepancy on the maximum diameter $D_{\max }=D_{b}+\Delta_{D}$ is less than $1 \%$. In the sequel, the laser sheet device is used for determining the above shape parameters.

The long time (over more than 100 'cardiac' periods) evolution of the maximum diameter for $K=40, L=1 \mathrm{~m}$ and $f=0.75 \mathrm{~Hz}$ is shown in figure $13(a)$ for various forcing amplitudes $a$. A threshold is visible: For amplitudes less than $90 \mathrm{~mm}$, the 

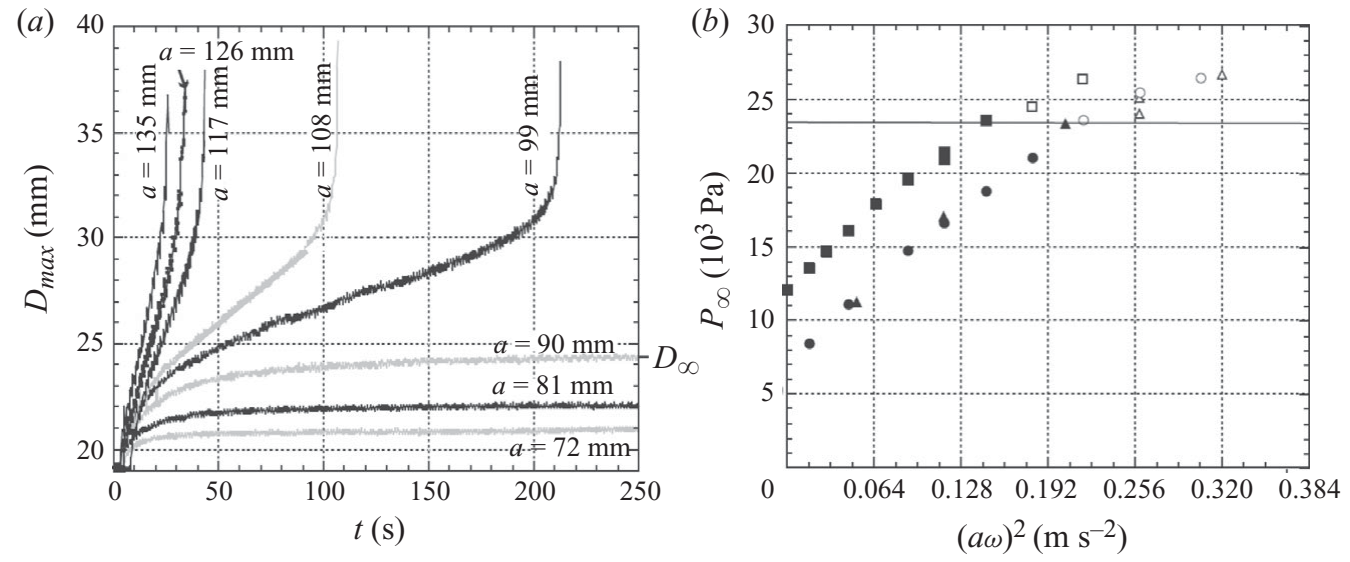

FIGURE 13. (a) Evolution of the maximum diameter as a function of time for various piston amplitudes $a ; K=40, L=1 \mathrm{~m}, f=0.75 \mathrm{~Hz}$. For amplitudes less than $90 \mathrm{~mm}$, the membrane reaches a stationary plateau, once the oscillations based on the cardiac cycle are filtered, whereas for amplitudes larger than $90 \mathrm{~mm}$, the maximum diameter diverges in finite time and an aneurysm is formed. $(b)$ Pressure reached in the membrane as a function of $(a \omega)^{2}$ for $K=40$ et $L=1 \mathrm{~m}$. In these experiments, the hydrostatic pressure has been varied by varying $H$. The evolution is presented for various combinations of forcing amplitudes and frequencies:

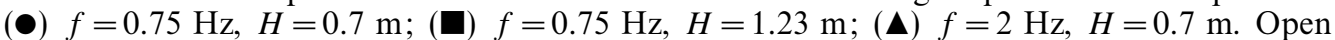
symbols correspond to experiments where an aneurysm forms. The horizontal line shows the maximum pressure in the curve $p(R)$.

membrane reaches a stationary plateau, once the oscillations based on the cardiac cycle are filtered out, whereas for amplitudes larger than $90 \mathrm{~mm}$, the maximum diameter diverges in finite time. Below the threshold, a characteristic settling time $t_{\infty}$ of a few dozen of cardiac cycles may be identified. Both $t_{\infty}$ and $D_{\infty}$ grow when the forcing amplitude is increased. Above the threshold, an inflexion point links the initial settling phase to a strongly growing stage.

Beyond the threshold, the cycle-averaged transmural pressure attains a steady value $p_{\infty}$ when the plateau regime is reached. This plateau pressure $p_{\infty}$ is shown as a function of $(a . \omega)^{2}$ in figure 13(b). Above the threshold, no plateau is reached, and since the experiment is stopped as soon as the aneurysm develops, the last measurement of the transmural pressure is retained and is shown (open symbols) in figure $13(b)$. The maximal pressure $p^{*}$ of the pressure-deformation law [defined in figure $4(b)$ ] is also shown. Since all pressures measured at an incipient aneurysm are above this value, whereas all plateau pressure values in the absence of any aneurysm are below this value, a clear correspondence may be drawn between the formation of an aneurysm and the appearance somewhere along the membrane of a pressure above $p^{*}$.

The forcing amplitude and the frequency at the threshold $\left(a_{c}, f_{c}\right)$ obtained by several similar experiments where the outlet hydraulic resistance $K$ and the membrane length $L$ are varied are shown together in figure 14. This figure clearly shows that the threshold amplitude $a_{c}$ is inversely proportional to the forcing frequency $f_{c}$, pointing to a threshold inflow rate. It further suggests that the membrane length $L$ does not influence the threshold and that larger hydraulic resistances $K$ favour aneurysms.

\subsection{Nonlinear Windkessel model for aneurysms}

As seen in figure 14, our experiments have shown that an aneurysm was triggered whenever the inlet flow rate $Q$ became higher than a certain threshold $Q^{*}$ increasing 


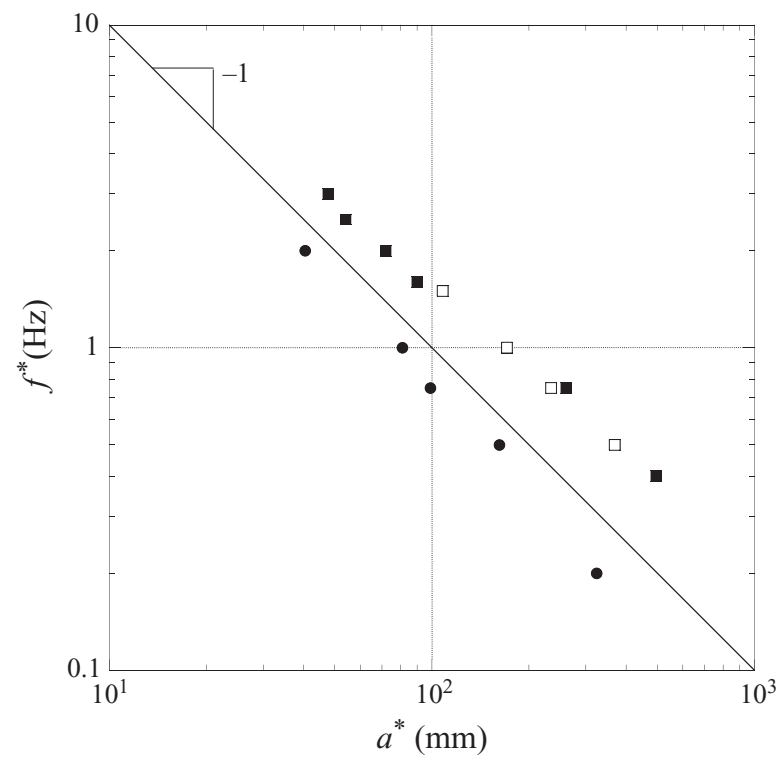

FIGURE 14. Amplitude and frequency threshold above which a bulge grows without bounds for several hydraulic outlet resistances and lengths. (•) $K=40, L=1 \mathrm{~m} ;(\boldsymbol{\square}) K=14, L=1 \mathrm{~m}$; ( $\square$ ) $K=14, L=0.5 \mathrm{~m}$.

with decreasing hydraulic resistance $K$ and independent of the length of the membrane. In this section, we derive a nonlinear Windkessel model accounting for this scaling.

The main difference with the study conducted in $\S 3.2$ is that we now consider large deformations so that the membrane law (2.3) is not linearized.

The outflow rate $Q_{\text {out }}$ is related to the pressure inside the membrane by (3.2) and thus gives

$$
Q_{\text {out }}(t)=\frac{A_{\text {out }} \sqrt{2} c_{0}}{\sqrt{K}} \sqrt{\frac{\sqrt{A / A_{0}}-1}{A / A_{0}}}
$$

Therefore, the Windkessel model becomes

$$
\frac{\mathrm{d} A}{\mathrm{~d} t}=\frac{Q_{\text {in }}(t)}{L}-\frac{A_{\text {out }} \sqrt{2} c_{0}}{\sqrt{K} L} \sqrt{\frac{\sqrt{A / A_{0}}-1}{A / A_{0}}} \equiv f(t, A) .
$$

In the present aneurysm regime, the deformations are large and the time scale $\tau_{e}$ characterizing the deformation of the membrane is large compared with the period of the forcing (in figure 13(a) $\omega \tau_{e} \sim 100$ and in physiology $\omega \tau_{e} \sim$ year s$^{-1} \sim 10^{7}$ ).

A multiple scale analysis can be followed, introducing the fast time scale $\tau=t / \epsilon$ with $\epsilon=1 / \omega \tau_{e}$, we write

$$
A=A^{(0)}(\tau, t)+\epsilon A^{(1)}(\tau)
$$

this yields

$$
\frac{1}{\epsilon} \frac{\partial A^{(0)}}{\partial \tau}+\frac{\partial A^{(0)}}{\partial t}+\frac{\partial A^{(1)}}{\partial \tau}=f\left(\tau, A^{(0)}+\epsilon A^{(1)}\right)=f\left(\tau, A^{(0)}\right)+O(\epsilon) .
$$


At order $1 / \epsilon, \partial A^{(0)} / \partial \tau=0$, meaning that the amplitude does not depend on the fast time scale. At leading order this amplitude is therefore the average over one period $(1 / T) \int_{0}^{T} A(t) \mathrm{d} t=A^{(0)}$. At the next order

$$
\frac{\partial A^{(1)}}{\partial \tau}=f\left(\tau, A^{(0)}\right)-\frac{\mathrm{d} A^{(0)}}{\mathrm{d} t} .
$$

If the average over one period of the right-hand side is not zero, it is easy to see that $A^{(1)}$ grows at least linearly. In that case, the initial separation between the amplitude and the fluctuation becomes invalid in a time of order $1 / \epsilon$. Therefore, necessarily

$$
\int_{0}^{T}\left(f\left(\tau, A^{(0)}\right)-\frac{\mathrm{d} A^{(0)}}{\mathrm{d} t}\right) \mathrm{d} \tau=0
$$

that is

$$
\frac{\mathrm{d} A^{(0)}}{\mathrm{d} t}=\frac{1}{T} \int_{0}^{T} f\left(\tau, A^{(0)}\right) \mathrm{d} \tau .
$$

With $f\left(\tau, A^{(0)}\right)$ given by (4.2), this yields

$$
\frac{\mathrm{d} A^{(0)}}{\mathrm{d} t}=\frac{1}{T L} \int_{0}^{T} Q_{\text {in }}(\tau) \mathrm{d} \tau-\frac{A_{\text {out }} \sqrt{2} c_{0}}{\sqrt{K} L} \sqrt{\frac{\sqrt{A^{(0)} / A_{0}}-1}{A^{(0)} / A_{0}}} .
$$

This equation can be further non-dimensionalized by introducing $A^{(0)}=A_{0} \bar{A}$, $t=\left((L \sqrt{K / 2}) / c_{0}\right) \bar{t}$ and $\bar{Q}_{i n}=\left(\sqrt{K / 2} / A_{0} c_{0}\right)(1 / T) \int_{0}^{T} Q_{i n}(\tau) \mathrm{d} \tau$, the reduced average incoming flow rate:

$$
\frac{\mathrm{d} \bar{A}}{\mathrm{~d} \bar{t}}=\bar{Q}_{i n}-\sqrt{\frac{\sqrt{\bar{A}}-1}{\bar{A}}} .
$$

Equation (4.9) shows that a steady state is attained $(\mathrm{d} \bar{A} / \mathrm{d} \bar{t}=0)$ only if the two terms on the right-hand side cancel. Since the function $\sqrt{(\sqrt{\bar{A}}-1) / \bar{A}}$ reaches the maximum value $1 / 2$ for $\bar{A}=4$ the balance is possible only if $\bar{Q}_{i n}<1 / 2$. Above this critical inflow, the amplitude grows without bound and enters the aneurysm regime.

This analysis is confirmed by the numerical integration of (4.9) performed in figure 15 for different values of $\bar{Q}_{i n}$. The two different behaviours (steady and aneurysm) are recovered and we also notice the similarity in the time evolution of the cross-section with the one measured experimentally (figure $13 a$ ).

A quantitative comparison of the non-dimensional threshold $\bar{Q}_{c}=1 / 2$ is provided in figure 16, with a remarkable agreement regarding the exact prefactor if one bears in mind the extreme simplicity of the Windkessel model considered here. Despite the two main limitations of the model, the time-averaging procedure resulting from the separation of scales and the global character of the model, which fully neglects the inhomogeneities described in $\S 3$, the agreement is excellent.

With dimensions, the critical mean flow $\bar{Q}_{c}=1 / 2$ above which aneurysms are expected is

$$
Q_{c}=A_{0} a_{c} \omega_{c}=\frac{A_{0} c_{0}}{\sqrt{2 K}}
$$




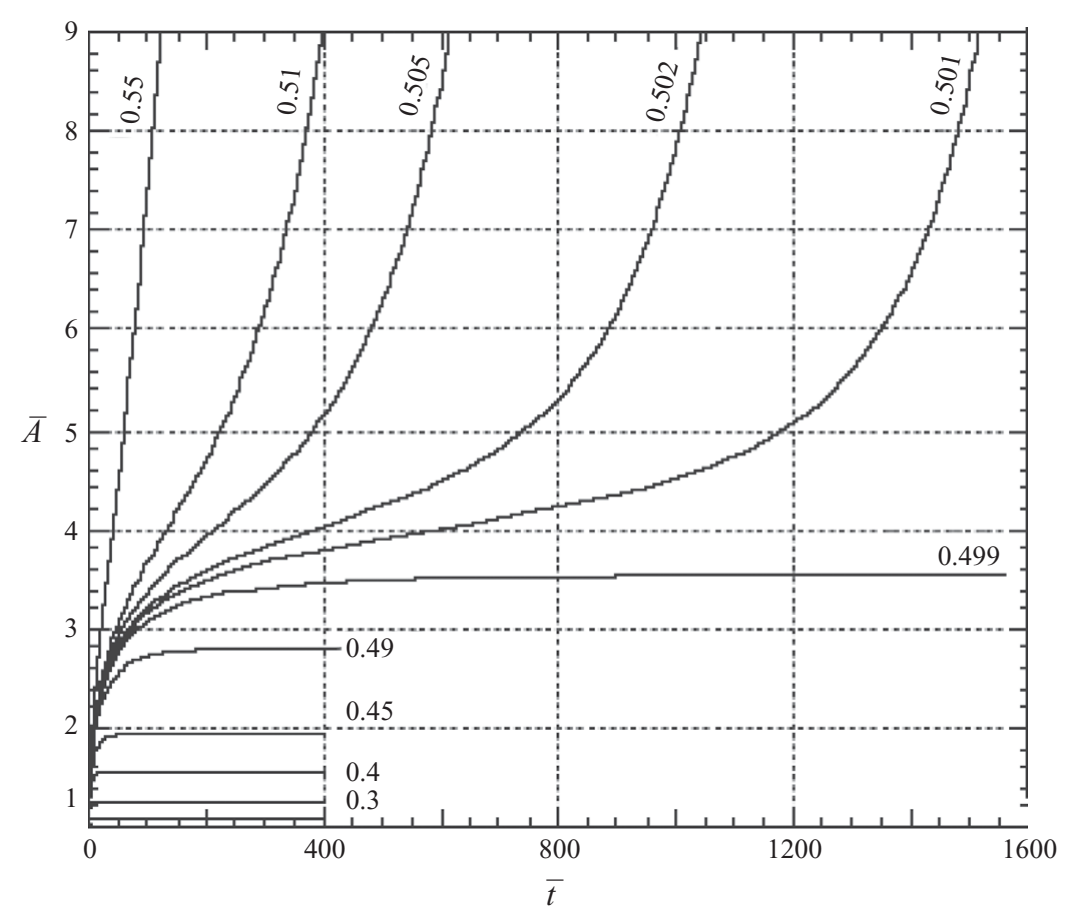

FIGURE 15. Theoretically predicted evolution of the mean cross-section as a function of non-dimensional time for various non-dimensional inflow rates. For non-dimensional inflow rates less than $1 / 2$, the membrane reaches a stationary plateau, whereas for inflow rates larger than $1 / 2$, the maximum cross-section diverges in finite time.

Under this form, the observations of figure 14 can be interpreted as follows:

(i) The threshold amplitude $a_{c}$ is inversely proportional to the forcing frequency $f_{c}$.

(ii) The membrane length $L$ does not influence the threshold.

(iii) Larger hydraulic resistances $K$ favour aneurysms.

\subsection{Numerical aneurysms experiments}

To go one step further the Windkessel model and discuss the localization of aneurysms, we perform a long-wave analysis of the flow in the spirit of Lighthill (1975) and get two conservation equations for mass and momentum:

$$
\begin{gathered}
\frac{\partial A}{\partial t}+\frac{\partial(A U)}{\partial x}=0 \\
\frac{\partial U}{\partial t}+\frac{\partial}{\partial x}\left[\frac{U^{2}}{2}+2 c_{0}^{2} \sqrt{A_{0}} \frac{\sqrt{A}-\sqrt{A_{0}}}{A}\right]=0
\end{gathered}
$$

A pulsating velocity is imposed at the entrance $(x=0): U(0, t)=\left(A_{0} / A\right) a \omega(1-$ $\cos \omega t) / 2$ and at the exit $(x=L) U(L, t)=p(L, t) / \mathscr{R}$, where the resistance $\mathscr{R}$ has the form $\mathscr{R}=\mathscr{R}_{0}+\rho K U(0, t)$. The first part is a constant $\mathscr{R}_{0}=10^{3} \mathrm{~Pa} \mathrm{~s} \mathrm{~m}^{-1}$. It accounts for the low-velocity regime where the resistance becomes of the Poiseuille type. This term is essential to ensure the numerical stability of the integration. The second part linear in $U$ reproduces the experimental trends observed for 'large' Reynolds numbers (figure 5). For a typical velocity $U=1 \mathrm{~m} \mathrm{~s}^{-1}$ and a resistance factor $K=10$, we find 


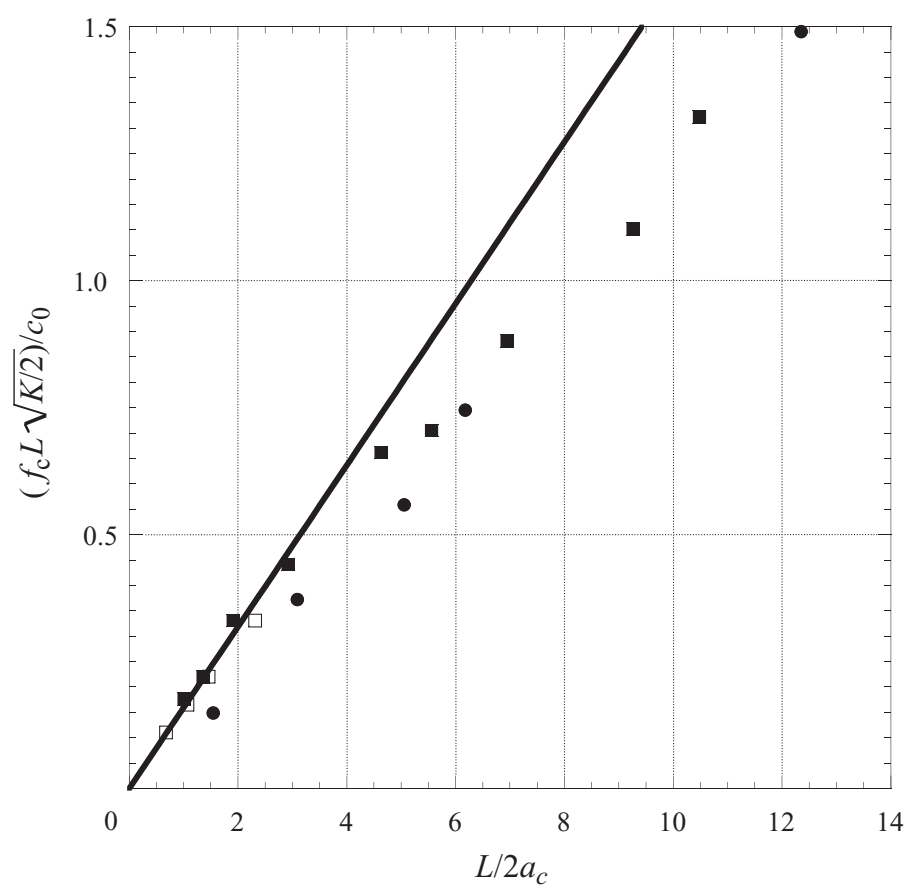

FIGURE 16. Non-dimensional critical frequency at the onset of an aneurysm $\bar{f}_{c}=\left((L \sqrt{K / 2}) / c_{0}\right) f_{c}$ as a function of $1 / 2 \bar{a}_{c}$, half the inverse of the non-dimensional critical amplitude $\bar{a}_{c}=a_{c} / L$ for the aneurysms reported in figure 14 . The predicted linear dependence with slope $1 / 2 \pi$ is shown by the solid line.

that the second component of the resistance is 10 times larger than the constant $\mathscr{R}_{0}$. The numerical resistance is thus close to that observed experimentally.

Equations (4.11) and (4.12) are solved using a finite-difference Lax-Friedrich scheme (see the Appendix) and a constant ratio $U /(\mathrm{d} x / \mathrm{d} t)=1 / 2$ satisfying the CourantFriedrichs-Lewy (CFL) condition. In this expression, $\mathrm{d} x$ and $\mathrm{d} t$ represent the space and time discretization intervals, respectively. As discussed by Hirsch (1989), this CFL condition is a necessary condition for convergence of the numerical integration.

In order to mimic the formation of aneurysms with our numerical model, we have observed that is was compulsory to introduce a more elastic region of the membrane, as shown in figure 17 , so as to prevent numerical instabilities at the outlet boundary condition. In the following, we have used a defect of width $\delta=L / 4$ (figure 17) and wave speed $c_{1}=c_{0} / \sqrt{2}$, located in the middle of the membrane. Note that the intensity of this defect could have been kept very low at the expense of a reduced time step. This in turn would have increased artificially the numerical dissipation of the Lax-Friedrich scheme and spuriously damped the dynamics.

Typical results are shown in figure 18 for a numerical membrane with $K=60$, $L=4 \mathrm{~m}, f=1 \mathrm{~Hz}$ and $c_{0}^{2}=35 \mathrm{~m}^{2} \mathrm{~s}^{-2}$. As in the experiment, two regimes are easily identified. For amplitudes less than $252 \mathrm{~mm}$, the membrane reaches a mean stationary plateau of the cross-section $A_{\infty}$ whereas for amplitudes larger than $252 \mathrm{~mm}$, the maximum diameter diverges in finite time. Again, beyond the threshold, a characteristic settling time $t_{e q}$ of a few dozen of cardiac cycles may be identified. 


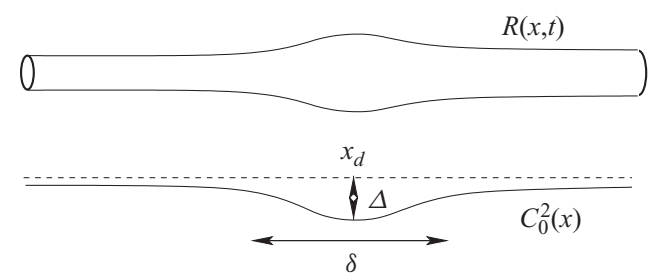

FIGURE 17. Typical inhomogeneous membrane properties used in the numerical experiments.

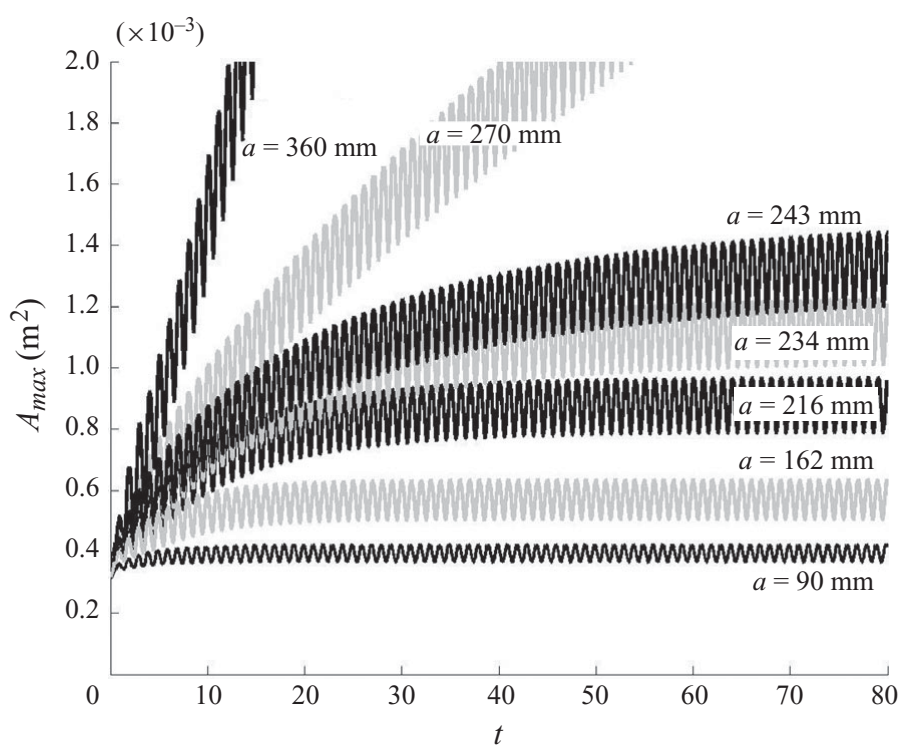

FIGURE 18. Evolution of the maximum cross-section as a function of time for various piston amplitudes $a ; K=60, L=4 \mathrm{~m}, f=1 \mathrm{~Hz}, c_{0}^{2}=35 \mathrm{~m}^{2} \mathrm{~s}^{-2}$. For amplitudes less than $252 \mathrm{~mm}$, the membrane reaches a stationary plateau once the oscillations based on the cardiac cycle are filtered, whereas for amplitudes larger than $252 \mathrm{~mm}$, the maximum cross-section diverges in finite time.

The numerical calculations are complementary to the experimental measurements, since they enable one to easily vary $L$ and $K$. A comparison of the threshold for unbounded growth obtained in the numerical model and the experiment is provided in figure 19 in the non-dimensional $1 / 2 \bar{a}_{c}-\bar{f}_{c}$ plane. Here, $\bar{f}_{c}=f_{c} L \sqrt{K / 2} / c_{0}$ and $\bar{a}_{c}=a_{c} / L$, in accordance with the previous section. The linear relationship, predicted by the nonlinear Windkessel model, is again verified here and it shows that aneurysms are formed whenever the flow rate $Q=A_{0} a \omega$ becomes larger than $Q_{c} \sim A_{0} c_{0} / \sqrt{2 K}$. There is however a discrepancy in the proportionality factor between the experiment and the numerical model. Several plausible interpretations may be proposed. The temporal flow rate signal in the numerical experiments differs from the experimentally imposed flow rate. Whereas a sinusoidal signal is used in the numerical integration, the experimental signal is more like a truncated sinus wave. It is likely that for a given amplitude and frequency, the peak experimental influx is larger than the numerical influx. Another difference lies in the fact that the membrane used in the numerical model does contain a localized defect. 


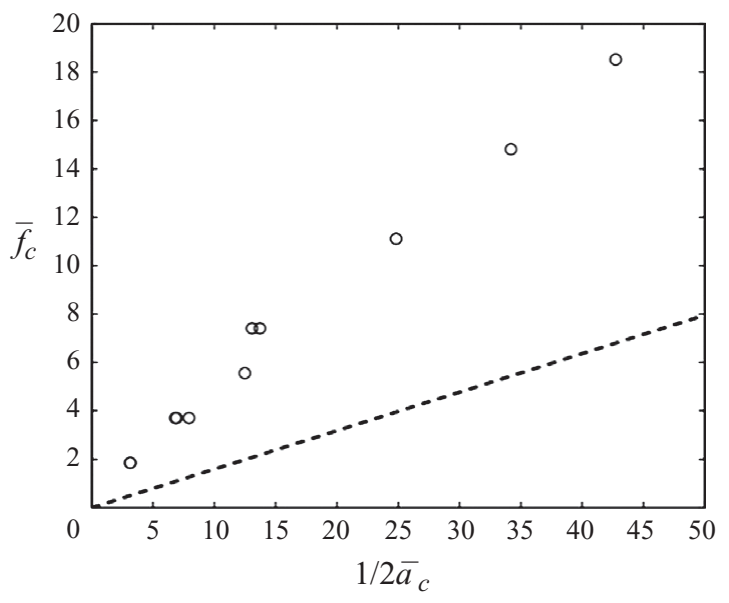

FIGURE 19. Non-dimensional threshold amplitude and frequency (denoted by open circles) for aneurysm in the numerical model (integration of the system (4.11) and (4.12)) for various lengths $L$ and hydraulic resistances $K$; the dashed line refers to the predicted linear dependence with slope $1 / 2 \pi(4.10)$.

\subsection{Influence of the nonhomogeneous deformations of the membrane onto the aneurysm threshold}

Section 3 was devoted to the analysis of various regimes of deformation of the membrane below the aneurysm threshold. It was shown that if the forcing time becomes smaller than the Windkessel settling time, the membrane could not fill and empty as a whole and becomes non-homogeneous. In the present section, we aim at considering the effect of such inhomogeneities onto the formation of aneurysm and to answer the question if they are particular regions in the membrane which encounter aneurysm at first as a consequence of focalization. From an experimental point of view, we have always observed aneurysms forming close to the end of the membrane, but we should remember that the length of the membrane could not be much varied, preventing a more systematic analysis. The numerical model should in principle enable a detailed study, but unfortunately it is not appropriate to yield a direct answer. Indeed, it is not possible to use a uniform membrane since in that case aneurysms would form at the end point and destabilize the boundary condition.

It is however possible to study the influence of the forcing-induced inhomogeneities in an indirect manner. For a given membrane, the position $x_{d}$ and the amplitude $\Delta=\left(c_{0}^{2}-c_{\min }^{2}\right) / c_{0}^{2}$ of the defect (see figure 17) can be varied until the aneurysm is triggered. Such a numerical experiment is shown in figure 20.

Figure 20(a1) shows that for the same defect intensity and width, the position of the centre of the defect has an influence on the aneurysm threshold. Indeed, for a defect located in the middle of the membrane $x_{d}=L / 2$, an aneurysm is formed, whereas for defects located upstream or downstream, the membrane reaches a mean equilibrium averaged shape shown in figure 20(a2). This time-averaged mean shape displays a bulge at the position of the defect, since the membrane is locally more distensible. The corresponding shape for $x_{d}=L / 2$ is not plotted since an aneurysm takes place, but for lower values of the defect intensity, the resulting bulge surpasses (not shown) those obtained for $x_{d}=L / 3$ and $x_{d}=2 L / 3$, and one may infer that, prior to aneurysm, the 
(a) $\left(\times 10^{-3}\right)$
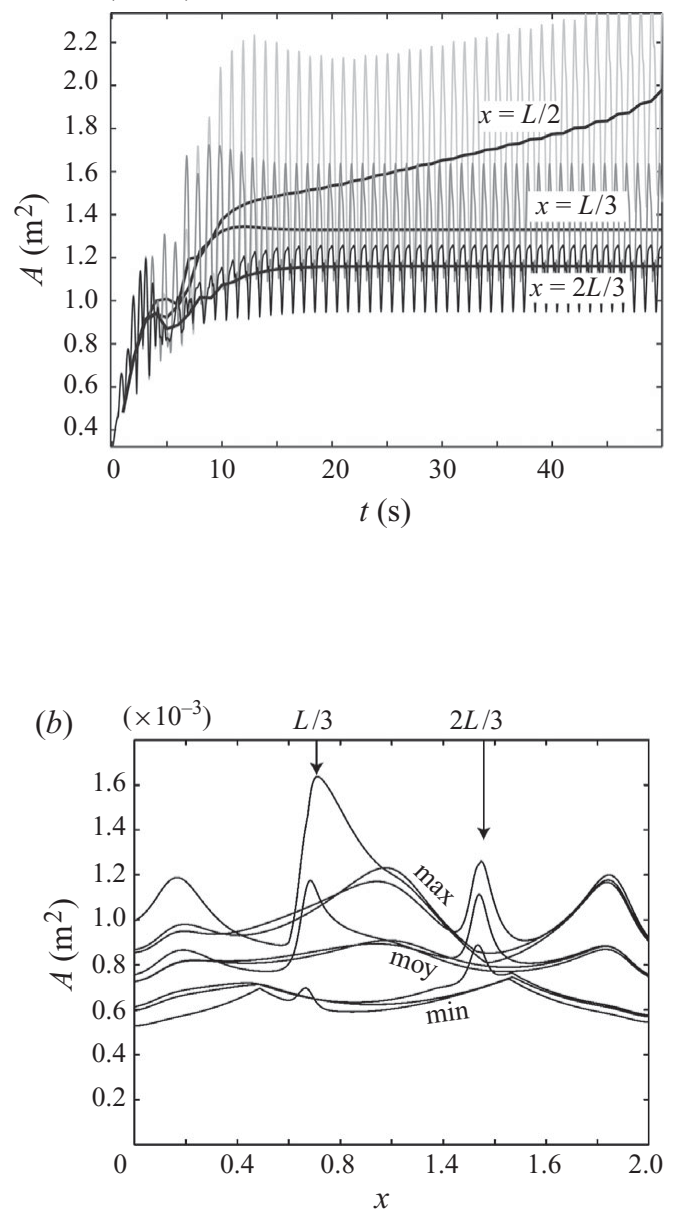

(c)

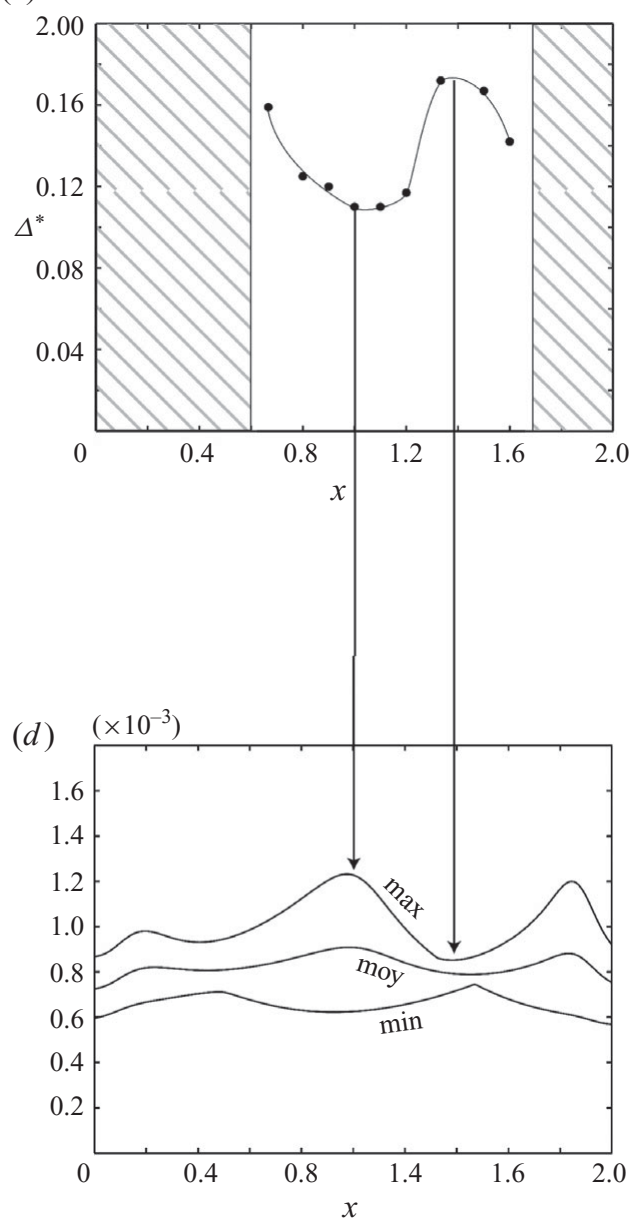

FIGURE 20. Numerical experiments on the influence of the position of the defect for a membrane with $L=2 \mathrm{~m}, f=1 \mathrm{~Hz}, a=360 \mathrm{~mm}, K=200$. (a) Time evolution of the maximal cross-section along the membrane, for three different positions of the same defect $(\Delta=0.12)$. (b) Maximal (max), minimal (min) and time-averaged (moy) mean cross-sections for an homogeneous membrane $(\Delta=0)$, and the two imperfect membranes at equilibrium: $x_{d}=L / 3, \Delta=0.12$ and $x_{d}=2 L / 3, \Delta=0.12$. (c) Threshold value of $\Delta^{*}$ required for the triggering of an aneurysm as a function of the position of the defect. The hashed zone could not be reached for numerical stability reasons. $(d)$ Maximal, minimal and time-averaged mean cross-sections for an homogeneous membrane $(\Delta=0)$, displaying highs and lows. The lowest $\Delta^{*}$ is obtained in the crest whereas the highest $\Delta^{*}$ is obtained in the trough.

maximum deformation with a localized defect increases with the mean deformation of the reference perfect membrane at the position of the defect. The influence of the defect location on the threshold for aneurysm is investigated quantitatively in figure 20(b1,b2), where the lowest required defect intensity is obtained for the position of the maximum focalization in the membrane without defect. Conversely, locations where the focalization is not active correspond to high threshold defect intensities. In this figure, positions of the defect close to the ends of the membrane could not be explored for numerical instability reasons. 


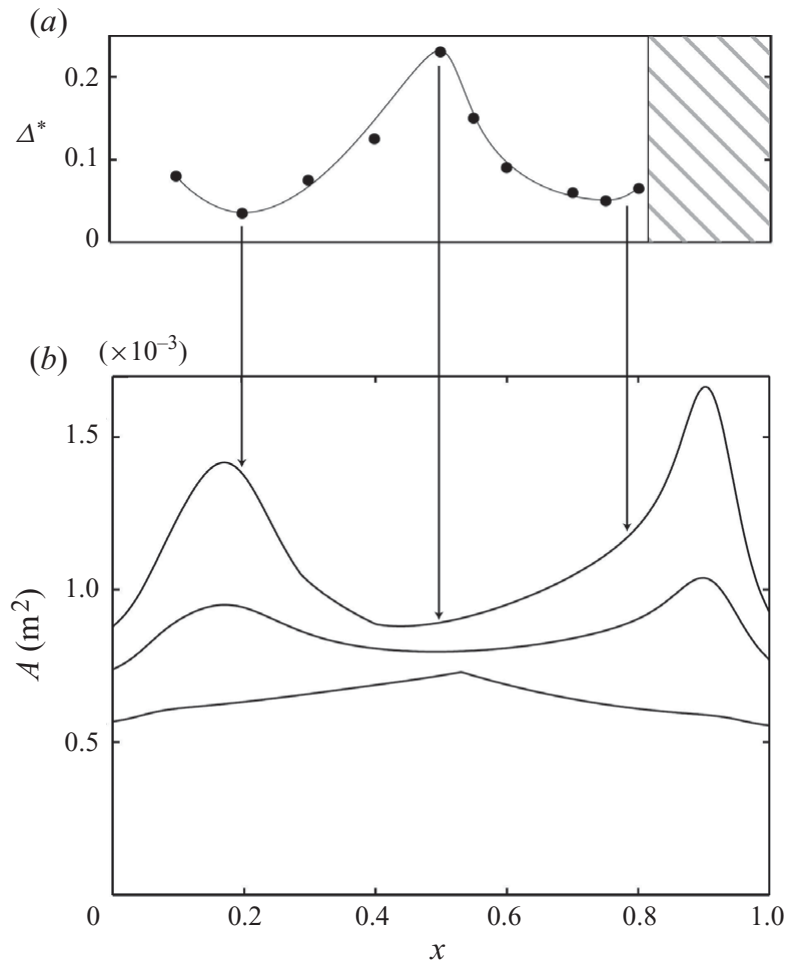

FIGURE 21. Numerical experiments on the influence of the position of the defect, similar to figure 20(b), with $L=1 \mathrm{~m}, f=1 \mathrm{~Hz}, a=360 \mathrm{~mm}, K=200$.

A similar analysis with the same trends is provided in figure 21 for another membrane length $L$. The lowest required defect intensity $\Delta^{*}$ is observed at the location of the reference membrane crests whereas the highest $\Delta^{*}$ is obtained in the trough. This confirms the influence of focalization onto the threshold for the formation of an aneurysm.

\subsection{Physiological implications}

Using formula (4.10) with a suitable prefactor to fit the experimental data and parameters suited to the aorta, $A_{0}=3 \times 10^{-4} \mathrm{~m}^{2}, c_{0}=5 \mathrm{~m} \mathrm{~s}^{-1}, K=10$, the threshold inflow becomes $Q_{c}=50 \mathrm{ml} \mathrm{s}{ }^{-1}$. This value is lower than the cardiac flux $80 \mathrm{ml} \mathrm{cycle}{ }^{-1}$, but note that only $50-70 \%$ of the flux goes into the aorta, the remaining flowing into the coronary and carotid arteries so that the order of magnitude of our threshold is close to the typical aortic blood flow rate.

Let us now discuss the possible link between our simple scaling law on the critical flow rate and the well-accepted risk factors for aneurysms:

(a) the age of the patient, inducing a rigidification of the aorta and other large arteries;

(b) hypertension, also associated with the rigidification of the aorta and augmentation of the mean and peak systolic pressure;

(c) atherosclerosis, i.e. the local deposition of lipidic calcified constituents leading to a stenosis;

(d) Marfan syndrome, a congenital disease that leads to a reduced elastin in the arteries wall. 
Regarding risk factors $(a)$ and $(b)$, the rigidification of iliac arteries leads to an increasing $K$, favouring aneursyms. The rigidification of the aorta however also leads to an increasing $c_{0}$, which should at first glance help to avoid aneurysms. However, the increased mean pressure produced by the heart to drive the flow through the more rigid membrane is associated with an increase in the peak diastolic pressure. This point needs to be explored in a subsequent study. Atherosclerosis $(c)$ may lead to a stenosis in the illiac increasing $K$. Alternatively, if the stenosis takes place in the aorta, the induced recirculation in the wake of the stenosis may lead to an enhancement of the shear in this lee region (Lasheras 2007), locally altering the mechanical properties of the aorta wall and ultimately weakening the elasticity properties in a finite region ( $\mathrm{Ku}$ et al. 1997). As shown in the preceding subsection, this weakened region is an excellent candidate for aneurysm formation. Finally, in the presence of Marfan syndrome $(d)$, the reduced elastin fraction leads to a lower $c_{0}$ that favours aneurysm formation.

\subsection{Further comments on the relevance of the study}

Note that the criterion (4.10) for aneurysm formation is based on the existence of a local maximum in the pressure law $P(R)$. In this paragraph, we refer to general features of membrane stability and use them to discuss the relevance of our results with respect to real aneurysms.

For a cylindrical membrane characterized by a pressure law $P(R)$ and filled with a liquid which moves from high- to low-pressure regions, it is straight forward to show that stable cylinders correspond to $\mathrm{d} P / \mathrm{d} R>0$ while membranes such that $\mathrm{d} P / \mathrm{d} R<0$ are unstable. A consequence of this remark is that capillary jets for which $P(R)=\gamma / R$ ( $\gamma>0$ represents the surface tension) are always unstable since $\mathrm{d} P / \mathrm{d} R=-\gamma / R^{2}$. This conclusion is correct in the long wavelength limit and is known as the SavartPlateau-Rayleigh instability (Savart 1833; Plateau 1849; Rayleigh 1879).

For an elastic membrane law such as (2.2), one finds $\mathrm{d} P / \mathrm{d} R=E d_{0} / R_{0}^{2}[(2-$ $\left.\left.R / R_{0}\right) /\left(R / R_{0}\right)\right]$. The membrane is thus expected to be stable in the range $R / R_{0}<2$ and to turn unstable above this critical value, as shown in $\S 4$. More generally, the existence of this stable to unstable transition is associated with the existence of a maximum in the $P(R)$ curve.

Now, let us show that the existence and typical geometry of aneurysms imposes the existence of such a maximum: since an aneurysm is a dilatation of a cylindrical artery which develops over the years, it does not evolve significantly on the time scale $\Delta \tau$ of few hundreds of heart beat cycles, and on this time scale, we can thus think of it as a steady deformation of the artery. Associating a mean pressure $\bar{p}$ with the mean flow, one can see that two states of different radii coexist in the membrane at the same mean pressure, similar to the case of an elastic membrane shown in figure 22(b). These two states $E_{1}$ and $E_{2}$ are reported in the general pressure-radius diagram in figure 22(a). These two states of different radii are stable on the time scale $\Delta \tau$ which imposes that $\mathrm{d} P / \mathrm{d} R$ is positive in $E_{1}$ and $E_{2}$. The segments $[A B]$ and $[C D]$ have thus positive slopes. Assuming that $P(R)$ is continuous, i.e. a slowly varying (in space and time) membrane law can be used, one deduces that the portion $[B C]$ will necessary contain a region of the negative slope. This shows the existence of a maximum between $E_{1}$ and $E_{2}$ which is at the origin of the shape instability which has led from the initial straight cylinder to the bulgy one.

For such an elastic membrane as we have used, this maximum originates directly in the cylindrical geometry and can be treated as a mechanical analogue of coexistent phases and treated with a Maxwell rule (Chater \& Hutchinson 1984a,b). For a real 

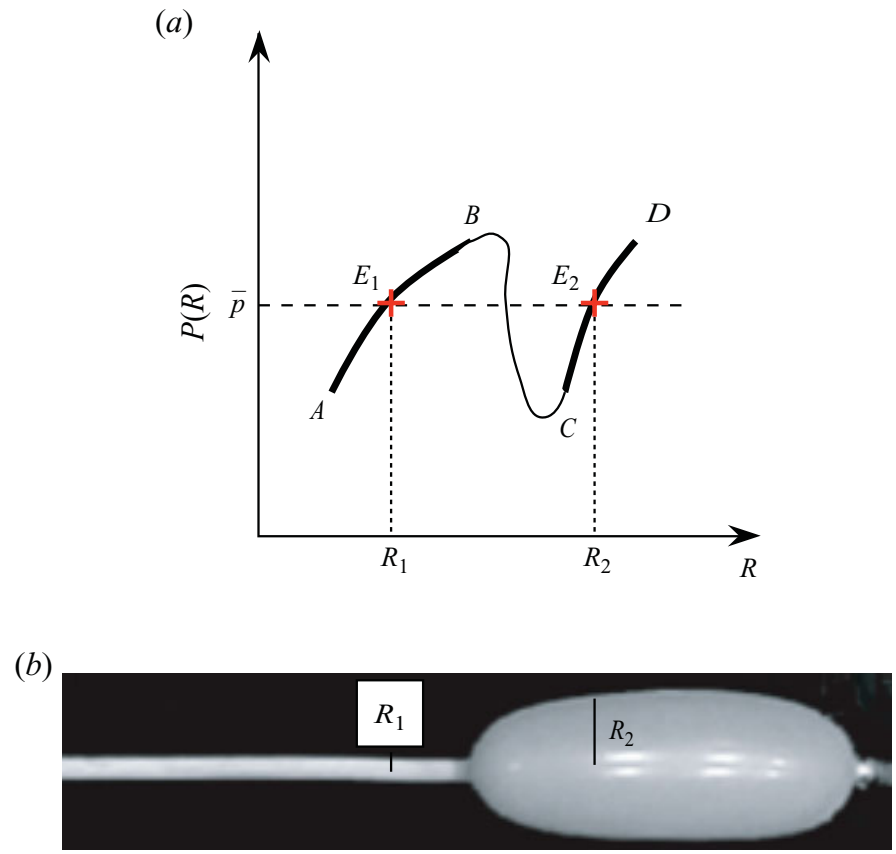

FIGURE 22. (Colour online) (a) General picture for the pressure-radius relation $P(R)$ on a membrane which presents a stationary dilatation $(b)$.

artery, the maximum has probably a different origin, resulting from the competition of geometrical and elastic nonlinearities. Still, as stressed above, the existence of aneurysms indicates that it should exist.

\section{Conclusion}

Abdominal aortic aneurysms are a dilatation of the aorta localized preferentially close to the bifurcation of the iliac arteries and which increases in time. Understanding their localization and growth rate remain two open questions which can have biological or physical origin or both. In order to identify the respective roles of the biological and physical processes, we have addressed these two questions of the localization and growth using a simplified physical experiment consisting of the pulsating flow in an elastic membrane in similarity with the flow in a human aorta.

We first show that submitted to an oscillating flow, the elastic membrane can undergo either an homogeneous deformation or an inhomogeneous deformation. We show that the transition between these two types of deformations is sensitive to both the frequency and the amplitude of the forcing. We propose a scaling argument to understand this transition and suggest a connection with the localization of real aneurysms.

Concerning the formation and development of bulges along the elastic membrane, we propose a simple model that captures the physics of the shape transition: in our elastic membrane, an 'aneurysm' forms whenever the time-averaged inflow is high enough for the time-averaged diameter of the membrane to double from its base value. At this specific dilatation, the relation between the inner pressure and the diameter presents a maximum, a property needed to observe the instability. 
The modifications brought to this global view by the influence of the local inhomogeneities in space has been tentatively discussed with the numerics and it is shown that the places where bulges develop are strongly related to the inhomogeneity of the deformation.

While discussing the possible application of this simple model to real aneurysms, we show that the critical flow rate criterion is compatible with most of known risk factors.

At this point, we have to underline that even if this simple model is promising, important biological factors such as the remodelling of the membrane properties have not been considered, and must be included in future work.

Similarly, we underline that in our experiments the time-dependent flow rate is prescribed, independent of the state of the latex tube or the downstream resistance. However, the heart does not exactly behave as a pure flow source, and if the peripheral resistance is changed, both the pressure and the flow pulses also change. This feedback loop will also be an interesting complement to the present model.

We thank B. Levy and A. Tedgui from INSERM U 541, Hôpital Lariboisière as well as P. Boutouyrie Inserm - U652 HEGP for the time they have allowed to discussions on the appropriate way to model aneurysms in a physics laboratory.

\section{Appendix. Detailed numerical scheme}

Equations (4.11) and (4.12) are discretized on a uniform finite difference grid with spacing $\mathrm{d} x$ according to

$$
\begin{gathered}
A_{n}^{p+1}=\frac{A_{n-1}^{p}+A_{n+1}^{p}}{2}+\frac{\mathrm{d} t}{2 \mathrm{~d} x}\left(U_{n+1}^{p} A_{n+1}^{p}-U_{n-1}^{p} A_{n-1}^{p}\right), \\
U_{n}^{p+1}=\frac{U_{n-1}^{p}+U_{n+1}^{p}}{2}+\frac{\mathrm{d} t}{2 \mathrm{~d} x}\left(\frac{\left(U_{n+1}^{p}\right)^{2}}{2}+c_{n+1}^{2} 2 \sqrt{\pi} R_{0} \frac{\sqrt{A_{n+1}^{p}}-\sqrt{A_{0}}}{A_{n+1}^{p}}\right. \\
\left.-\frac{\left(U_{n-1}^{p}\right)^{2}}{2} \cdots-c_{n-1}^{2} 2 \sqrt{\pi} R_{0} \frac{\sqrt{A_{n-1}^{p}}-\sqrt{A_{0}}}{A_{n-1}^{p}}\right),
\end{gathered}
$$

where $n$ refers to the spatial index, $p$ refers to the temporal index and $\mathrm{d} t$ is the time discretization interval. Note that in these equations $c_{0}$ has been replaced by $c(x)$, a function of $x$, which enables us to consider the membrane with non-uniform elasticity. The limit conditions used at the entrance are

$$
\begin{aligned}
A_{1}^{p+1} & =A_{1}^{p}+\frac{\mathrm{d} t}{\mathrm{~d} x}\left(U_{2}^{p} A_{2}^{p}-U_{1}^{p} A_{1}^{p}\right), \\
U_{1}^{p+1} & =\frac{D^{p+1}}{A_{1}^{p}},
\end{aligned}
$$

where $D^{p+1}=D(0, t=(p+1) \delta t)=A_{0} a \omega(-1+\cos [\omega(p+1) \delta t]) / 2$. At the exit we impose

$$
\begin{aligned}
& A_{N}^{p+1}=A_{N}^{p}+\frac{\mathrm{d} t}{\mathrm{~d} x}\left(U_{N}^{p} A_{N}^{p}-U_{N-1}^{p} A_{N-1}^{p}\right), \\
& U_{N}^{p+1}=-2 \rho c_{N}^{2} \sqrt{\pi} R_{0} \frac{\sqrt{A_{N}^{p}}-\sqrt{A_{0}}}{\mathscr{R} A_{N}^{p}},
\end{aligned}
$$

with $\mathscr{R}=\mathscr{R}_{0}+\rho K U(0, t)$. 


\section{REFERENCES}

AleXANder, J. J. 2004 The pathobiology of aortic aneurysms. J. Surg. Res. 117, 163-175.

Carpenter, P. W. \& Pedley, T. J. 2003 Flow in Collapsible Tubes and Past Other Highly Complaint Boundaries. Kluwer.

Chandran, K. B. \& Yearwood, T. L. 1981 Experimental study of physiological pulsatile flow in a curved tube. J. Fluid Mech. 111, 59-85.

Chater, E. \& Hutchinson, J. W. 1984a On the propagation of bulges and buckles. J. Appl. Mech. 51, 269-277.

Chater, E. \& Hutchinson, J. W. $1984 b$ Mechanical analogs of coexistent phases. In Phase Transformations and Material Instabilities in Solids, pp. 21-36. Academic Press Inc. ISBN 0-12-309770-3.

DE Chauliac, G. 1373 La grande chirurgie (ed. C. Michel). Imprimeur de l'Université de Montpellier.

Cheng, C. P., Herfokens, R. J. \& TaYlor, C. A. 2003 Abdominal aortic hemodynamic conditions in healthy subjects aged 50-70 at rest and during lower limb exercise: in vivo quantification using MRI. Atherosclerosis 168, 323-331.

Frank, O. 1905 Der Puls in den Arterien. Z. Biol. 45, 441-553.

Fung, Y. C. 1990 Biomechanics: Motion, Flow, Stress and Growth. Springer.

FunG, Y. C. 1997 Biomechanics: Circulation. Springer.

Gray, H. 1918 Anatomy of the Human Body. Lea and Febiger.

Glagov, S., Rowley, D. A. \& Kohut, R. 1961 Atherosclerosis of human aorta and its coronary and renal arteries. Arch. Pathol. Lab. Med. 72, 558-568.

Groenink, M., Langevaka, S. E., Vanbavel, Ed., van der Wall, E. E., Mulder, B. J. M., van der WaL, A. C. \& SpaAn, J. A. E. 1999 The influence of aging and aortic stiffness on permanent dilation and breaking stress of the thoracic descending aorta. Cardiovasc. Res. 43, 471-480.

Guirguis, E. M. \& Barber, G. G. 1991 The natural history of abdominal aortic aneurysms. Am. J. Surg. 162, 481-483.

Hirsch, C. 1989 Numerical Computation of Internal and External Flows. Wiley.

Humphrey, J. D. \& Delange, S. L. 2004 An Introduction to Biomechanics (Solids and Fluids, Analysis and Design). Springer.

Ku, D. N. 1997 Blood flow in arteries. Annu. Rev. Fluid Mech. 29, 399-434.

Ku, D. N., Zeigler, M. N. \& Downing, J. M. 1990 One-dimensional steady inviscid flow through a stenotic collapsible tube. J. Biomech. Engng 112, 444-450.

Laennec, R. T. H. 1819 De l'auscultation Médiate ou Traité du Diagnostic des Maladies des Poumons et du Coeur, Fondé Principalement Sur ce Nouveau Moyen d'exploitation. J. A. Brosson \& J. S. Chaud.

Lasheras, J. C. 2007 The biomechanics of arterial aneurysms. Annu. Rev. Fluid Mech. 39, 293-319.

Li, J. K., Malbin, J., Riffle, R. A. \& Noodergraaf, A. 1981 Pulse wave propagation. Circ. Res. 49, 442-452.

Lighthill, J. 1975 Mathematical Biofluiddynamics. SIAM.

McAuley, L. M., Fisher, A., Hill, A. B. \& Joyce, J. 2002 Les Implants Endovasculaires Comparativement à la Chirurgie Sanglante Dans la Réparation de L'anévrisme de L'aorte Abdominale: Pratique au Canada et Examen Systématique. Rapport Technologique no. 33. Office canadien de coordination de l'évaluation des technologies de la santé.

McDonald, D. A. 1960 Blood Flow in Arteries. Edward Arnold.

McDonald, D. A. 1968 Regional pulse-wave velocity in the arterial tree. J. Appl. Physiol. 24, 73-78.

Medynsky, A. O., Holdsworth, D. W., Sherebrin, M. H., Rankin, R. N. \& Roach, M. R. 1998 Elastic response of human iliac arteries in-vitro to balloon angioplasty using high-resolution CT1. J. Biomech. 31, 747-751.

Olsen, J. H. \& Shapiro, A. H. 1967 Large amplitude unsteady flow in liquid-filled elastic tubes. J. Fluid Mech. 29, 513-538.

Pä̈Doussis, M. P. 2006 Wave propagation in physiological collapsible tubes and a proposal for a Shapiro number. J. Fluids Struct. 22, 721-725.

Paquerot, J. F. \& Lambrakos, S. G. 1994 Monovariable representation of blood flow in a large elastic artery. Phys. Rev. E 49, 3432-3439.

Pedley, T. J. 1980 The Fluid Mechanics of Large Blood Vessels. Cambridge University Press. 
Plateau, J. A. F. 1849 Statique expérimentale et théorique des liquides soumis aux seules forces moléculaires. Acad. Sci. Brux. Mem. 23, 5.

Prandtl, L. \& Tietuens, O. G. 1957 Applied Hydro and Aeromechanics. Dover.

Rayleigh, Lord. 1879 On the instability of jets. Proc. Lond. Math. Soc. 10, 4-13.

Reinke, W., Johnson, P. C. \& Gaehtgens, P. 1986 Effect of shear rate variation on apparent viscosity of human blood in tubes of 29 to 94 microns diameter. Circ. Res. 59, 124-132.

Roberts, J. C., Moses, C. \& Wilkins, R. H. 1847 Autopsy studies in atherosclerosis: distribution and severity of atherosclerosis in patients dying without any morphologic evidence of atherosclerotic catastrophe. Circulation 20, 511-519.

SAVART, F. 1833 Mémoire sur la constitution des veines liquides lancées par des orifices circulaires en mince paroi. Ann. de Chim. 53, 337-386.

ShapIro, A. H. 1977 Steady flow in collapsible tubes. ASME J. Biomech. Engng 99, 126-147.

WomersLey, J. R. 1957 Oscillatory flow in arteries: the constrained elastic tube as a model of arterial flow and pulse transmission. Phys. Med. Biol. 2, 178-187. 